\title{
El territorio de Tonina, Chiapas
}

Le territoire de Tonina, Chiapas

The Tonina territory, Chiapas

\section{Eric Taladoire}

\section{OpenEdition}

\section{Journals}

Edición electrónica

URL: https://journals.openedition.org/jsa/15200

DOI: 10.4000/jsa. 15200

ISSN: 1957-7842

\section{Editor}

Société des américanistes

\section{Edición impresa}

Fecha de publicación: 15 diciembre 2017

Paginación: 141-173

ISSN: 0037-9174

\section{Referencia electrónica}

Eric Taladoire, «El territorio de Tonina, Chiapas», Journal de la Société des américanistes [En línea], 103-2 | 2017, Publicado el 15 diciembre 2017, consultado el 26 septiembre 2022. URL: http:// journals.openedition.org/jsa/15200 ; DOI: https://doi.org/10.4000/jsa.15200

All rights reserved 


\title{
El territorio de Tonina, Chiapas
}

\author{
Eric TALADOIRE *
}

\begin{abstract}
Durante los últimos treinta años, las investigaciones desarrolladas tanto en Tonina como en las localidades vecinas modifican nuestras interpretaciones de la ocupación humana del valle de Ocosingo. Autorizan una revisión de la cronología con la posible identificación de una fase de transición del Clásico Terminal, consecutiva a la existencia de relaciones entre los habitantes del valle y sus vecinos de los Altos de Chiapas. Esos lazos tal vez hayan permitido a una parte de los habitantes del valle sobrevivir por algún tiempo al colapso maya. [Palabras clave: valle de Ocosingo, patrón de asentamiento, élites, territorio, Clásico Terminal.]
\end{abstract}

Le territoire de Tonina, Chiapas. Les recherches de ces trente dernières années, tant à Tonina que dans les régions voisines, modifient la validité de nos interprétations sur l'occupation humaine de la vallée d'Ocosingo. Cette relecture conduit à une révision de la chronologie, avec l'identification potentielle d'une phase de transition datée du Classique Terminal, qui résulterait de l'existence de liens entre les habitants de la vallée et leurs voisins des terres hautes. Ces liens ont peut-être permis à une partie des habitants de la vallée de surmonter quelque temps l'effondrement maya. [Motsclés : vallée d'Ocosingo, habitat, élites dirigeantes, territoire, Classique Terminal.]

The Tonina territory, Chiapas. In the light of the investigations of these last thirty years, at Tonina as well as in neighbouring regions, it is possible to modify our previous interpretations of the Ocosingo valley occupation. They allow for a revision of the chronological frame and the identification of a potential transition phase during the Terminal Classic, related to existing relations between the valley inhabitants and their neighbours from the Chiapas Highlands. These relations possibly allowed part of the valley inhabitants to survive for some time the Maya collapse. [Key words: Ocosingo valley, settlement pattern, elites, territory, Terminal Classic.]

La ciudad de Tonina se ubica en las márgenes occidentales del área maya (Figura 1). El valle de Ocosingo ocupa una posición intermedia entre las tierras bajas, donde florecieron las grandes ciudades clásicas, y las tierras altas de

* Archéologie des Amériques, université Paris 1 Panthéon-Sorbonne [taladoire@yahoo.fr]. 
Chiapas, todavía mal conocidas a pesar de múltiples investigaciones recientes (Álvarez 1993; Laló Jacinto y Aguilar 1993; Paris, Taladoire y Lee 2013, 2015). Caracterizada por su estilo escultórico específico en piedra arenisca, su afición por la guerra y por el sacrificio de cautivos, Tonina suele ser considerada como una ciudad menor que otras capitales mayas; pero, a pesar de su aislamiento y de sus particularidades, sabemos que estaba intensamente involucrada en los acontecimientos históricos de la civilización maya (Martin y Grube 2000). Sin embargo, falta mucho para comprender su papel exacto y evaluar la forma en que este sitio se ubica en la evolución del área maya.

Descrito por primera vez en 1840 por Stephens y Catherwood, el sitio fue objeto luego de numerosas visitas (Blom y LaFarge 1926-1927; Blom y Duby 1955-1957; Thompson 1944, por ejemplo), las cuales enriquecieron el corpus de esculturas y permitieron la identificación de algunos sitios menores como Pestac. En 1971, P. Becquelin y C. Baudez elaboraron un proyecto arqueológico que fue desarrollado durante varias temporadas (1971-1973, 1977 y 1979-1981). Los resultados de esas excavaciones se publicaron hace casi treinta años (Becquelin, Baudez [dir.] 1979, 1982; Becquelin, Taladoire [dir.] 1990). Salvo una breve intervención en 1993 (Becquelin, Michelet y Taladoire 1994), las investigaciones de la Misión Arqueológica Francesa en el valle de Ocosingo resultan entonces remotas.

Una parte importante de las actividades de la Misión Francesa estuvo dedicada al reconocimiento sistemático del valle de Ocosingo y a las excavaciones de diversos sitios secundarios (1979-1981). Desde 1982, Yadeun (1992, 1993) ha trabajado continuamente en Tonina y sus investigaciones proporcionaron numerosos datos, tanto arquitectónicos como escultóricos, que amplían nuestra comprensión de la historia del sitio. No hay todavía en sus publicaciones datos relativos a investigaciones en otros sitios del valle, por lo que los obtenidos previamente permanecen vigentes.

Los estudios cerámicos recientes (Rands, Bishop y Sabloff 1982; Demarest, Rice P. y Rice D.S. 2004; Forsyth 2005; Munõz 2006; Forné et al. 2010), así como los resultados de las últimas excavaciones (Yadeun 1992, 1993, com. pers. 2008) que evidenciaron actividades constructivas en Tonina luego de su destrucción ${ }^{1}$, confirman que una parte importante de la población del valle de Ocosingo no sólo sobrevivió sino que trató de revitalizar la ciudad. El conjunto de datos disponible por el momento permite sugerir la existencia de una ocupación relativamente densa durante el Clásico Terminal (840-1000 d.C.), que no había sido posible postular previamente. En efecto, al momento de

1. Las excavaciones de Yadeun en la parte alta de la Acrópolis documentaron fosas con numerosos vestigios óseos, que interpreta como indicios de una expedición guerrera que terminó con gran parte de la élite de la ciudad y que probablemente haya destruido varios monumentos (Yadeun 1993, p. 33). Todavía no se han publicado los datos. 
establecer la secuencia cerámica de Tonina (Becquelin y Baudez 1979-1982) el concepto de Clásico Terminal estaba todavía muy mal definido (Demarest, Rice P. y Rice D.S. 2004), lo cual fue una de las razones para asignar a la fase Ixim una duración de hasta 910 d.C. (Figura 1).

La definición de la entidad política de Tonina, de sus aliados y sus satélites ha sido el foco de otro artículo (Taladoire 2015). Aquí tratamos de esbozar la evolución del patrón de asentamiento del valle de Ocosingo y sus implicancias para la definición del territorio de Tonina, basándonos en los resultados obtenidos a lo largo de las diversas temporadas de excavación.

\begin{tabular}{|c|c|c|}
\hline Periodos & $\begin{array}{c}\text { Becquelin y Baudez } \\
(1979-1982)\end{array}$ & Propuesta cronológica \\
\hline Preclásico Tardío & Wach $(0-300$ d.C. $)$ & Wach $(0-300$ d.C. $)$ \\
\hline Clásico Temprano & May $(330-550$ d.C. $)$ & May $(330-550$ d.C. $)$ \\
\hline Clásico Tardío & Ixim $(600-910$ d.C. $)$ & Ixim $(600-840$ d.C. $)$ \\
\hline Clásico Terminal & Ixim $(600-910$ d.C. $)$ & $\begin{array}{c}\text { Ixim-Chenek } \\
(840-1000 \text { d.C. })\end{array}$ \\
\hline Posclásico Temprano & Chenek $(1000-1250$ d.C. $)$ & Chenek $(1000-1250$ d.C. $)$ \\
\hline Posclásico Tardío & Chib $(1250-1528$ d.C. $)$ & Chib $(1250-1528$ d.C. $)$ \\
\hline
\end{tabular}

Fig. 1 - Esquema cronológico revisado de la secuencia de Tonina.

De entrada debemos subrayar el carácter relativo de los datos y de las interpretaciones que se presentan a continuación. A pesar de ser sistemáticos, los reconocimientos de superficie sólo proporcionan datos parciales en ausencia de excavaciones, aun cuando 50 de los 128 sitios registrados en el valle han sido objeto de sondeos estratigráficos. Además, muchas partes del territorio de la entidad política de Tonina no han sido objeto de investigaciones (Taladoire 2015, Figura 2, ver página siguiente). Varios sitios de la región occidental del área maya aguardan ser investigados detalladamente (Schele 1991). No se sabe casi nada de Xoc (Ekholm 1973) o de Santa Elena Poco Uinic (Palacios 1928), salvo una nota de Mathews (sitio Wayeb s.f.). Se conoce todavía muy poco sobre Bonampak y Plan de Ayutla-Sak T'zi (Anaya Hernández, Guenter y Zender 2003).

Además, el valle de Ocosingo presenta dos dificultades complementarias que interfirieron con la investigación (Figura 3, ver página siguiente). Las faldas sur-oeste y norte del valle cuentan con aflojamientos de piedra caliza dura que dieron pie a formas naturales regulares que se confunden fácilmente con vestigios antrópicos como montículos o basamentos piramidales. Cinco de ellos se identificaron como posibles sitios y sólo excavándolos pudieron descontarse del corpus (Becquelin, Michelet y Taladoire 1994). Por otra parte, en el siglo pasado, los suelos fértiles y el clima templado de esa misma falda suroeste y de los alrededores del pueblo de Sivaca favorecieron el desarrollo del cultivo de caña y de una producción de azúcar en ingenios domésticos. En 
Eric TALADOIRE

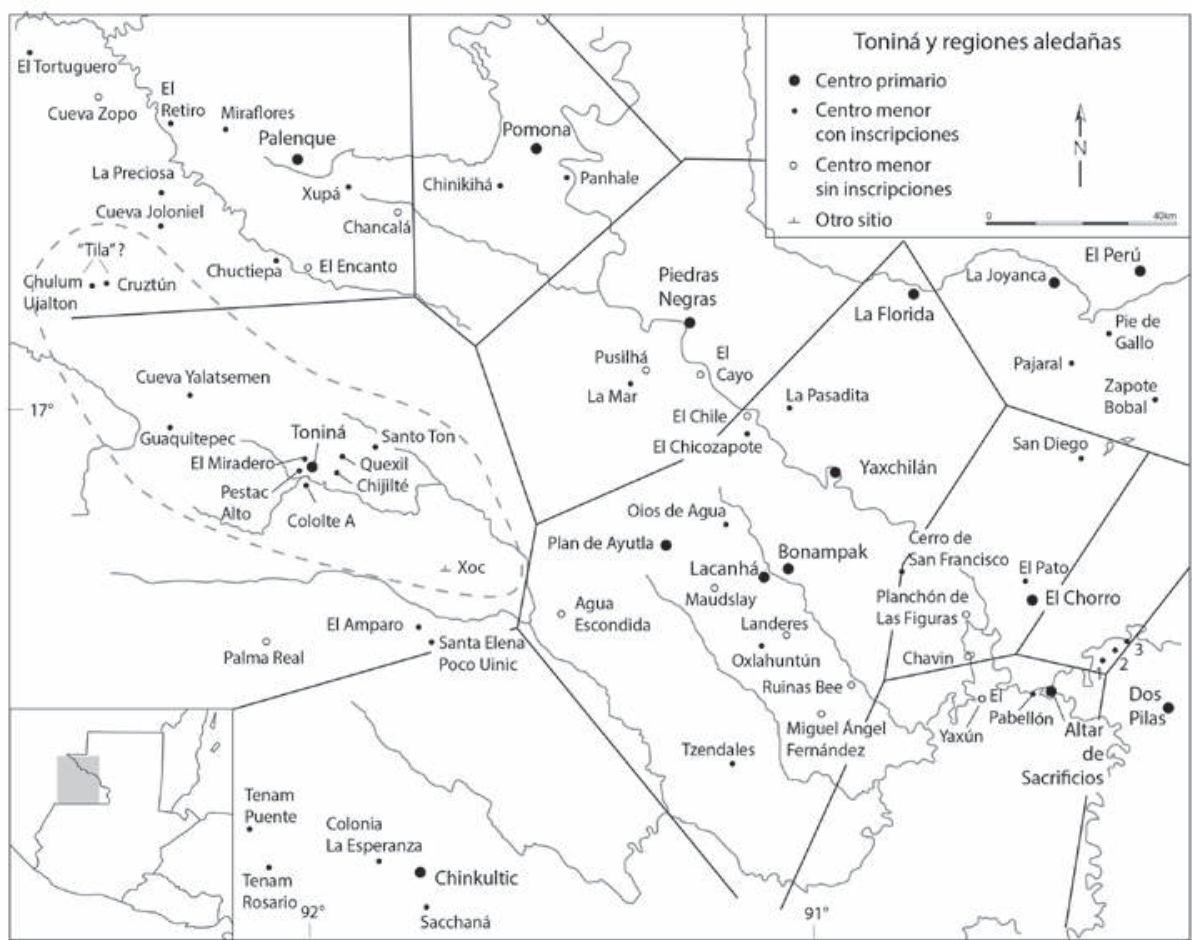

Fig. 2 - Tonina y su territorio en 9.13.0.0.0. (Taladoire 2015, dib. Sylvie Éliés).

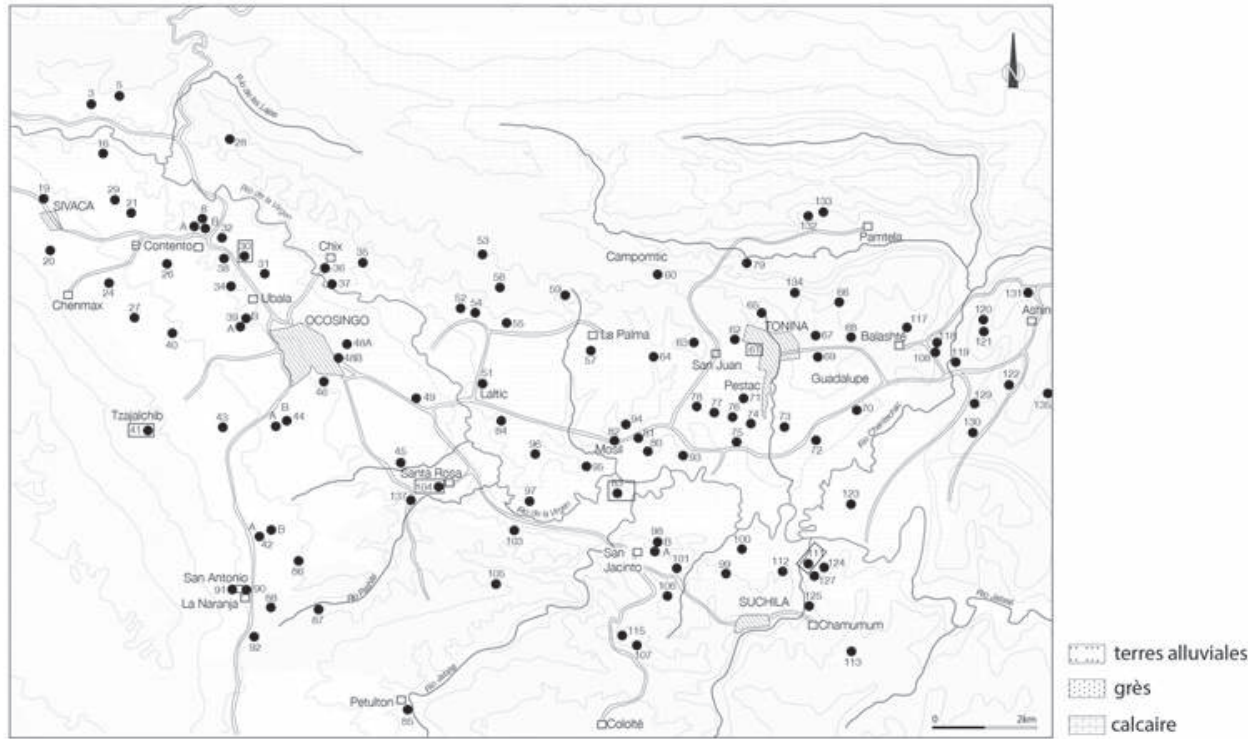

Fig. 3 - Mapa geomorfológico del valle de Ocosingo (según Usselman, dib. Sylvie Éliés). 
el reconocimiento preliminar se registraron 28 loci como posibles sitios sobre la base de la presencia de grandes cantidades de tepalcates en superficie y de arreglos antrópicos. Sólo el análisis de la cerámica permitió determinar que el tipo Sivaca Burdo, parecido al tipo Cololté Incensario del Clásico Tardío, correspondía a dicha actividad azucarera (Becquelin, Taladoire [dir.] 1990, p. 1622-1624). Al finalizar el reconocimiento, entonces, se suprimieron del inventario por presentar únicamente material de ese tipo. Pero siempre existe la posibilidad de que algunos de ellos hayan sido ocupados anteriormente, en la época prehispánica. Ante la ausencia de excavaciones intensivas, pues, nuestras conclusiones no pueden ser más que provisionales ${ }^{2}$.

Para compensar lo relativo del reconocimiento, resulta indispensable entonces recurrir a otros datos procedentes de los estudios geográficos, estilísticos y epigráficos. Cada uno de ellos enriquece nuestra comprensión; siempre, como veremos, con sus propios límites. La suma de datos relativos no proporciona una percepción absoluta, pero al menos nos permite realizar un acercamiento global a lo que era el territorio de Tonina en el valle de Ocosingo.

\section{Marco geográfico}

De forma ovalada (24 por 5.5 kilómetros), el valle de Ocosingo está constituido por el curso medio del río Jataté y de sus afluentes, el Chantechac y el río de la Virgen (Figura 3). Su parte central es plana, dividida entre zonas inundables y terrazas más altas, entre dos cadenas de lomas areniscas sobre las cuales se ubica la mayoría de los asentamientos prehispánicos, Tonina incluido (Usselmann en Becquelin, Taladoire [dir.] 1990, p. 1491-1501). Un pequeño valle secundario, separado por esas lomas, se ubica inmediatamente al norte del valle principal. En la falda suroeste, el macizo de piedra caliza dura del anticlinal Nazareth domina directamente el valle.

A unos cinco kilómetros al este de Tonina las cadenas de lomas areniscas se juntan cerrando la extremidad oriental del valle, mientras que, al oeste, el valle se vuelve más estrecho, aunque abierto hacia el curso superior del Jataté. El valle donde se desarrollaron los reconocimientos constituye entonces una unidad geográfica bien delimitada, con verdaderos límites naturales. Considerando la importancia del curso superior del Jataté, Becquelin (Becquelin y Baudez 1982) procedió en 1977 a un reconocimiento complementario de la región de ChilónBachajón, que comparte con el valle de Ocosingo los mismos rasgos físicos naturales (Figura 4, ver página siguiente).

El valle de Ocosingo se encuentra a unos $900 \mathrm{msnm}$, en la zona fronteriza entre los Altos de Chiapas y las tierras bajas. El clima resulta relativamente cálido (24을 de temperatura promedio), con una media anual de $1800 \mathrm{~mm}$ de

2. El total de sitios suprimidos del inventario preliminar alcanza entonces 33. 


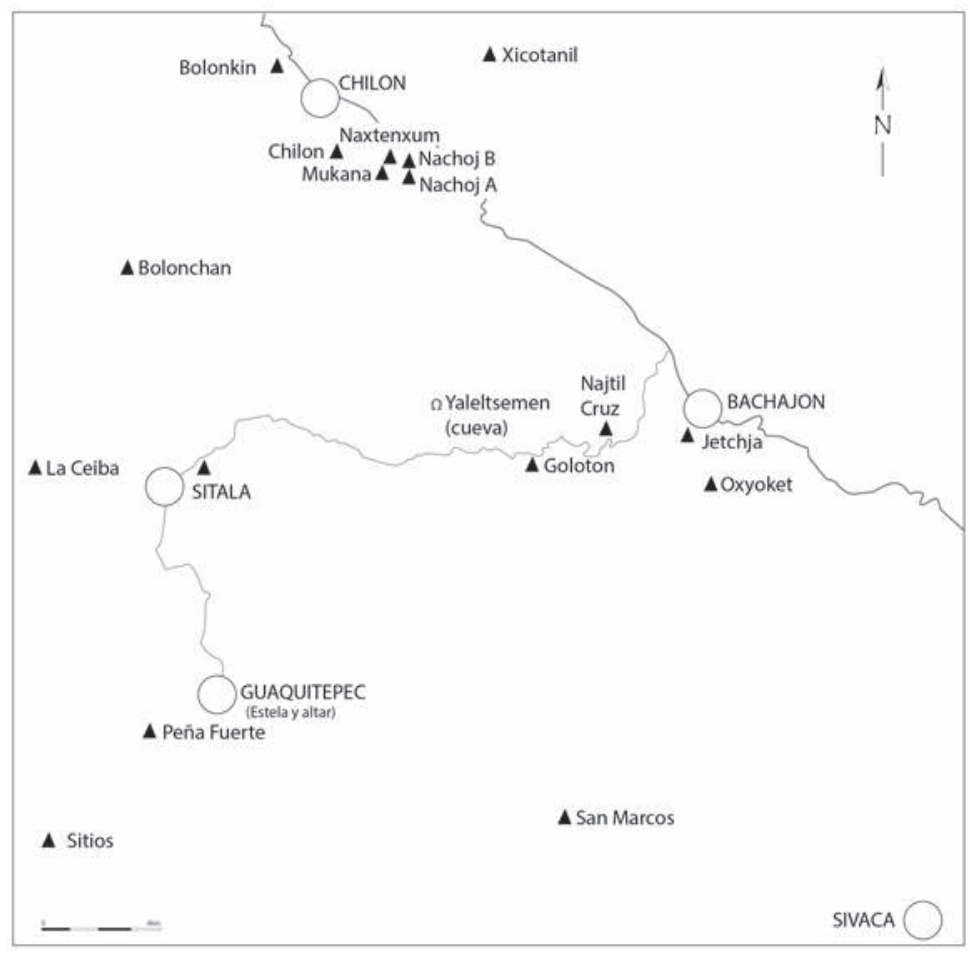

Fig. 4 - La región de Chilón-Bachajón y los sitios documentados por Becquelin (1977) (dib. Sylvie Éliés).

precipitaciones. Los ríos permanentes proporcionan recursos hidráulicos y proveen un flujo continuo de aluviones que conforman suelos fértiles. El valle dispone así de recursos florísticos muy variados, desde una vegetación tropical perennifolia en el fondo (caña, plátanos, algodón) hasta una vegetación boreal (robles, pinos) en las vertientes (Puig en Becquelin, Taladoire [dir.] 1990, p. 1491-1501). Así, el valle de Ocosingo dispone de los recursos específicos de las tierras altas, ausentes en los otros sitios de las tierras bajas. La coincidencia de suelos fértiles, clima tropical y abundancia de agua permite el desarrollo en el valle de actividades agrícolas productivas sin recurrir a métodos intensivos como terrazas o irrigación (Taladoire 2016). No se registraron ejemplos de tales acondicionamientos en el reconocimiento ni tampoco en las investigaciones geomorfológicas y botánicas (Becquelin, Taladoire [dir.] 1990).

A pesar de su ubicación en la tradición cultural de las tierras bajas, el valle de Ocosingo se diferencia entonces de sus vecinos por su acceso inmediato a medioambientes muy distintos. El valle constituye una entidad geográfica bien definida, con límites naturales que lo definen pero no lo aíslan. El curso inferior del río Jataté abre un acceso relativamente fácil hacia el valle del Usumacinta, 
y su curso superior hacia los valles de Chilón-Bachajón. La presencia en la vertiente meridional del valle de las tierras altas de Chiapas facilita a su vez el acceso directo a los Altos, y aun una posible entrada a enemigos potenciales. Sólo las elevaciones de la Sierra Norte de Chiapas forman una barrera montañosa al norte que dificulta las relaciones con el noreste (Golden et al. 2012).

\section{Los reconocimientos y el patrón de asentamiento}

Al empezar las excavaciones de Tonina, en 1972, ya se conocían varios sitios menores (Laltic, Pestac, Santo Ton, Quexil/San Ricardo) gracias a los trabajos de investigadores previos (Blom y LaFarge 1926-1927; Blom y Duby 19551957; Thompson 1944 por mencionar los principales).

Puesto que las excavaciones de las dos primeras temporadas se enfocaron en Tonina, los reconocimientos resultaron reducidos (Becquelin, Baudez [dir.] 1982). Si bien se visitó el conjunto global del valle, incluyendo el valle norte ${ }^{3}$, sólo se registraron 41 sitios con vestigios mayores (pirámides, monumentos) entre los más representativos de las distintas fases de ocupación: Mosil C y Tonina Norte para el Preclásico Tardío (fase Wach), Santa Teresa para el Clásico Temprano (fase May), Chamumún (Clásico Terminal) y Tzajalchib (fase Chib) para el Posclásico Tardío. Se hicieron excavaciones en seis de los sitios mayores representativos de cada fase. En Tzajalchib sólo fue posible una recolección de superficie, complementando los datos obtenidos por Culbert (1965) ${ }^{4}$.

El estilo de las esculturas de Guaquitepec se parece al de Tonina y sus inscripciones mencionan su glifo-emblema (Mayer 1980, 1984), lo que sugiere la pertenencia de la región de Chilón-Bachajón a la entidad de Tonina. Basándose en esos datos, en 1977 Becquelin efectuó un reconocimiento complementario en esta zona (Becquelin y Baudez 1982). Además de tres sitios del Posclásico Temprano, Becquelin registró un total de seis sitios del Clásico Tardío, con material característico de la fase Ixim en el sitio de San Marcos (Fig. 3). Así, consideramos confirmada la pertenencia de esta zona a la esfera política de Tonina (Becquelin, Baudez [dir.] 1979; Andrieu et al. 2011; Sheseña Hernández y Tovalín-Ahumada 2014; Taladoire 2015).

Éramos conscientes de las fallas de los reconocimientos anteriores: el número de sitios identificados resultaba bajo y casi no se habían registrados grupos residenciales menores. Por otro lado, la ocupación documentada para el Preclásico Tardío parecía importante, con un sitio grande (Mosil C) y otro más pequeño (Tonina Norte), pero con uno de los pocos juegos de pelota preclásicos por

3. Becquelin efectuó excavaciones en Tonina Norte.

4. En las últimas temporadas, se hicieron dos pozos estratigráficos en Tzajalchib. 


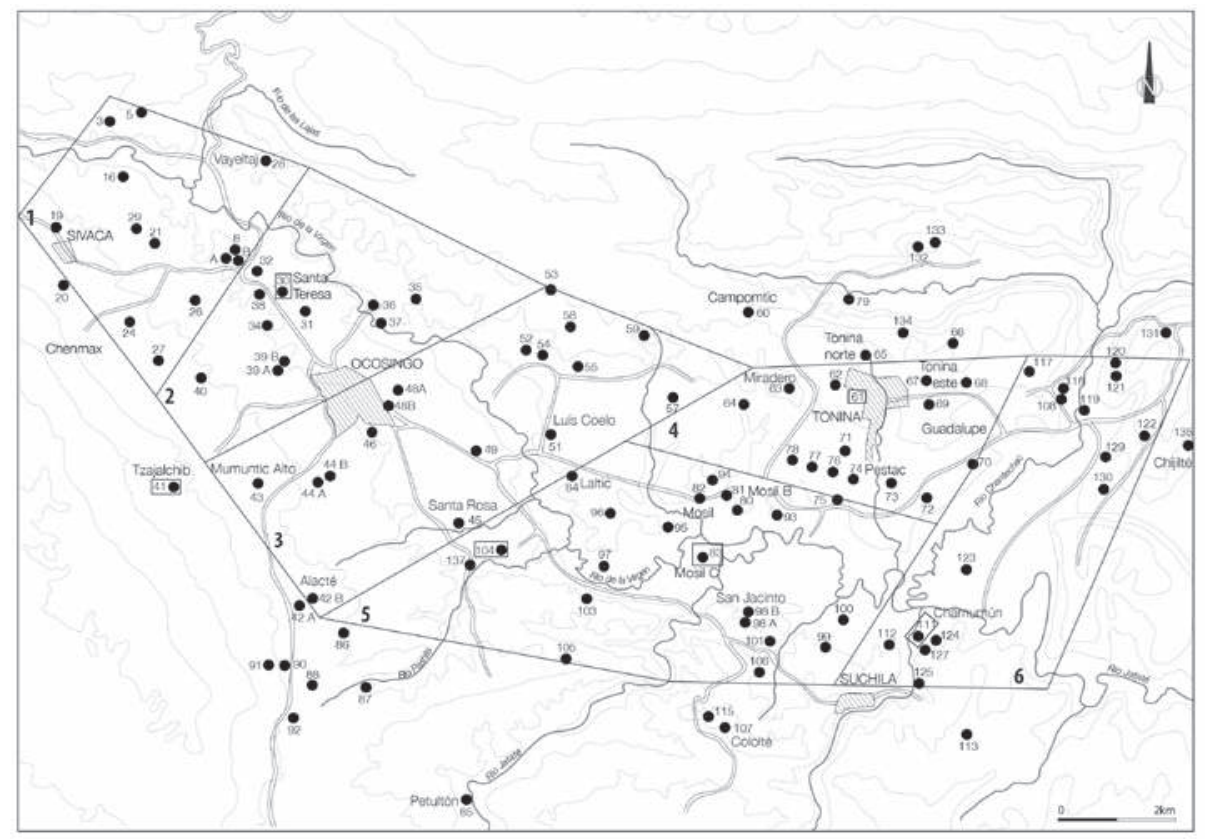

Fig. 5 - Mapa general de la ocupación prehispánica del valle de

Ocosingo, y zonas de reconocimiento (dib. Sylvie Éliés).

entonces conocidos 5 . A la inversa, la fase May del Clásico Temprano estaba mal representada con una sola ocupación documentada en Santa Teresa. Las temporadas de 1979 y 1980 fueron dedicadas al reconocimiento sistemático del valle (Figura 5), o sea $102 \mathrm{~km}^{2}$ (Becquelin, Taladoire [dir.] 1990). Faltó tiempo para el reconocimiento del valle anexo norte.

El valle se dividió en seis zonas de manera arbitraria (Figura 5), y en 1979 un reconocimiento preliminar cubrió la parte central, registrando 29 sitios. En 1980 se recogió todo el valle. Gracias a un informante se identificaron sitios secundarios al sur de las zonas de reconocimiento. En la mayoría de los sitios se raspó sistemáticamente una capa de $10 \mathrm{~cm}$ para recoger tepalcates. Se registraron 57 sitios suplementarios, llegando así al total de 128 sitios potenciales. Como ya se mencionó, varios de ellos no se corresponden con ocupaciones prehispánicas, ya sea porque resultaron ser formaciones naturales de piedra caliza ${ }^{6}$, o bien por el carácter reciente de las actividades (los ingenios). De esos 128 asentamientos, 50 fueron objeto de pozos estratigráficos. En Mosil B, una cala permitió

5. Al momento de la publicación, sólo se conocían los juegos de pelota preclásicos tardíos de Cerros (Scarborough 1991).

6. Se trata de los sitios n. ${ }^{\text {os }} 1,4,43,114$ y 116 , todos ubicados en el vertiente sur del valle. 
confirmar la existencia de una cancha de juego de pelota (Taladoire 1980). Varias veces, por ejemplo en Vayeltaj, las excavaciones ubicaron en niveles inferiores vestigios arquitectónicos que sugieren la presencia de estructuras anteriores. Sobre la base de las excavaciones y las recolecciones de superficie, fue posible fechar 96 sitios y asentamientos ${ }^{7}$ (Figura 6). Regresaremos más adelante a la interpretación de los resultados, pero es necesario hacer hincapié en el último reconocimiento llevado a cabo en 1993 (Becquelin, Michelet y Taladoire 1994).

Fig. 6 - Lista de los sitios fechados, en orden cronológico (E: excavaciones, $\mathrm{S}$ : superficie), con su posición en las zonas de reconocimiento (Z) y su ubicación (U) en las orillas derecha (D) o izquierda (I) del Río Jataté. (A): Arquitectura, (E): Estela.

\begin{tabular}{|c|c|c|c|c|c|c|c|c|c|c|c|}
\hline & Sitio & & Wach & May & Ixim & Chenek & Chib & $\mathbf{Z}$ & $\mathbf{U}$ & $\mathbf{A}$ & $\mathbf{E}$ \\
\hline 61 & Tonina & E & $\mathrm{X}$ & $X$ & $X$ & $\mathrm{X}$ & $X$ & 4 & I & $\mathrm{X}$ & $X$ \\
\hline 36 & Chix & $\mathrm{E}$ & $\mathrm{X}$ & & $\mathrm{X}$ & $\mathrm{X}$ & & 2 & $\mathrm{I}$ & & \\
\hline 65 & Tonina Norte & $\mathrm{E}$ & $\mathrm{X}$ & & & & & 4 & I & $X$ & \\
\hline 80 & Jocotaj & $\mathrm{E}$ & $\mathrm{X}$ & & $\mathrm{X}$ & & & 5 & I & $\mathrm{X}$ & \\
\hline 83 & Mosil C & $\mathrm{E}$ & $\mathrm{X}$ & & $\mathrm{X}$ & & & 5 & $\mathrm{D}$ & $\mathrm{X}$ & $\mathrm{X}$ \\
\hline 84 & Laltic & $E$ & $X$ & & $\mathrm{X}$ & $\mathrm{X}$ & & 5 & I & $X$ & $\mathrm{X}$ \\
\hline 104 & $\begin{array}{l}\text { Rancho de } \\
\text { Santa Rosa }\end{array}$ & $\mathrm{E}$ & $\mathrm{X}$ & & $\mathrm{X}$ & $\mathrm{X}$ & & 5 & $\mathrm{D}$ & $\mathrm{X}$ & \\
\hline 123 & Chantechac sur & E & X & & & & & 6 & I & & \\
\hline 30 & Santa Teresa & $\mathrm{E}$ & & $X$ & & $\mathrm{X}$ & $X$ & 2 & $\mathrm{D}$ & $X$ & \\
\hline 67 & Tonina Este & $\mathrm{E}$ & & $\mathrm{X}$ & $X$ & & & 4 & I & $\mathrm{X}$ & $\mathrm{X}$ \\
\hline 72 & Guadalupe sur & $\mathrm{S}$ & & $\mathrm{X}$ & & & & 4 & I & & \\
\hline 29 & Las Rocas & $\mathrm{E}$ & & & $X$ & & & 1 & D & & \\
\hline 32 & La Ladrillera & $\mathrm{E}$ & & & $\mathrm{X}$ & & & 2 & $\mathrm{D}$ & & \\
\hline 35 & Tuminteel & $\mathrm{S}$ & & & $\mathrm{X}$ & & & 2 & I & & \\
\hline 37 & $\begin{array}{l}\text { Potrero del } \\
\text { Mariposal }\end{array}$ & $\mathrm{S}$ & & & $\mathrm{X}$ & & & 2 & I & & \\
\hline 45 & Santa Rosa & $\mathrm{S}$ & & & $X$ & & & 3 & D & & \\
\hline 48 & Campo Aéreo & $\mathrm{E}$ & & & $\mathrm{X}$ & & & 3 & $\mathrm{D}$ & & \\
\hline 49 & Pasilha & $\mathrm{E}$ & & & $\mathrm{X}$ & & & 3 & $\mathrm{D}$ & & \\
\hline 51 & Luís Coelo & $\mathrm{E}$ & & & $\mathrm{X}$ & & & 3 & I & $\mathrm{X}$ & $\mathrm{X}$ \\
\hline 52 & Coelo & $\mathrm{S}$ & & & $\mathrm{X}$ & & & 3 & I & & \\
\hline 53 & Valladolid & $\mathrm{S}$ & & & $\mathrm{X}$ & & & 3 & I & & \\
\hline 55 & $\begin{array}{c}\text { Rancho Joaquín } \\
\text { Millares }\end{array}$ & $\mathrm{E}$ & & & $\mathrm{X}$ & & & 3 & I & & \\
\hline 62 & El Sacramento & $\mathrm{S}$ & & & $\mathrm{X}$ & & & 4 & $\mathrm{I}$ & & $\mathrm{X}$ \\
\hline 66 & Buenavista & $\mathrm{E}$ & & & $\mathrm{X}$ & & & 4 & I & & \\
\hline 68 & $\begin{array}{l}\text { Guadalupe } \\
\text { Oeste }\end{array}$ & $\mathrm{E}$ & & & $X$ & & & 4 & I & & \\
\hline
\end{tabular}

7. Entre los sitios fechados figuran unos ingenios recientes. 
Eric Taladoire

\begin{tabular}{|c|c|c|c|c|c|c|c|c|c|}
\hline 70 & Guadalupe & $\mathrm{S}$ & $X$ & & & 4 & I & & \\
\hline 73 & Wuluxbak & $\mathrm{S}$ & $\mathrm{X}$ & & & 4 & I & $\mathrm{X}$ & \\
\hline 74 & Pestac Alto & $\mathrm{S}$ & $\mathrm{X}$ & & & 4 & I & & $\mathrm{X}$ \\
\hline 75 & $\begin{array}{l}\text { Rancho José } \\
\text { Solórzano }\end{array}$ & $\mathrm{E}$ & $X$ & & & 4 & I & & \\
\hline 76 & Ehcalumil & $\mathrm{S}$ & $\mathrm{X}$ & & & 4 & I & & \\
\hline 77 & Luís Gordillo & $\mathrm{S}$ & $\mathrm{X}$ & & & 4 & I & & \\
\hline 82 & Mosil A & $\mathrm{E}$ & $X$ & & & 5 & I & & \\
\hline 90 & $\begin{array}{l}\text { San Antonio } \\
\text { La Naranja }\end{array}$ & $\mathrm{S}$ & $X$ & & & 5 & $\mathrm{D}$ & & \\
\hline 91 & $\begin{array}{l}\text { Rancho El } \\
\text { Naranjo }\end{array}$ & $\mathrm{E}$ & $X$ & & & 5 & $\mathrm{D}$ & & \\
\hline 97 & El Naranjo & $\mathrm{S}$ & $\mathrm{X}$ & & & 5 & I & & \\
\hline 105 & Chiclaianhuitz & $\mathrm{S}$ & $\mathrm{X}$ & & & 5 & $\mathrm{D}$ & & \\
\hline 119 & $\begin{array}{l}\text { Chantechac } \\
\text { Norte }\end{array}$ & $\mathrm{E}$ & $X$ & & & 6 & I & & \\
\hline 121 & Ashin sur & $\mathrm{S}$ & $\mathrm{X}$ & & & 6 & I & & \\
\hline 122 & $\begin{array}{c}\text { Ashin Campo } \\
\text { Redondo }\end{array}$ & $\mathrm{E}$ & $X$ & & & 6 & I & & \\
\hline 129 & $\begin{array}{c}\text { Las } \\
\text { Chachalacas }\end{array}$ & $\mathrm{E}$ & $X$ & & & 6 & I & & \\
\hline 130 & Chanoa & $\mathrm{S}$ & $\mathrm{X}$ & & & 6 & I & & \\
\hline 134 & La Libertad & $\mathrm{S}$ & $\mathrm{X}$ & & & 4 & I & & \\
\hline 137 & El Saqueo & $\mathrm{S}$ & $X$ & & & 5 & $\mathrm{D}$ & & \\
\hline 142 & $\begin{array}{l}\text { San Vicente } \\
\text { Fracción }\end{array}$ & $\mathrm{S}$ & $X$ & & & 5 & I & & \\
\hline 145 & $\begin{array}{l}\text { Campomtic } \\
\text { Oeste }\end{array}$ & $\mathrm{S}$ & X & & & & I & & \\
\hline 7 & Contento & $\mathrm{S}$ & $\mathrm{X}$ & $\mathrm{X}$ & & 1 & $\mathrm{D}$ & & \\
\hline 24 & $\begin{array}{l}\text { Chenmax San } \\
\text { Luís }\end{array}$ & $\mathrm{E}$ & $\mathrm{X}$ & $\mathrm{X}$ & & 1 & $\mathrm{D}$ & & \\
\hline 28 & Vayeltaj & $\mathrm{E}$ & $\mathrm{X}$ & $\mathrm{X}$ & & 1 & I & & \\
\hline 63 & Miradero & $\mathrm{E}$ & $\mathrm{X}$ & $\mathrm{X}$ & & 4 & $\mathrm{I}$ & & \\
\hline 81 & Mosil B & $\mathrm{E}$ & $\mathrm{X}$ & $\mathrm{X}$ & & 5 & $\mathrm{I}$ & $\mathrm{X}$ & \\
\hline 96 & El Hormiguero & $\mathrm{S}$ & $X$ & $\mathrm{X}$ & & 5 & I & & $\mathrm{X}$ \\
\hline 107 & Cololté A & $\mathrm{S}$ & $\mathrm{X}$ & $X$ & & 5 & $\mathrm{D}$ & & $\mathrm{X}$ \\
\hline 111 & Chamumún & $\mathrm{E}$ & $\mathrm{X}$ & $\mathrm{X}$ & & 6 & $\mathrm{D}$ & $\mathrm{X}$ & \\
\hline 127 & Chakham & $\mathrm{E}$ & $\mathrm{X}$ & $X$ & & 6 & $\mathrm{D}$ & & \\
\hline 8 & El Aserradero & $\mathrm{E}$ & $\mathrm{X}$ & $X$ & $\mathrm{X}$ & 1 & $\mathrm{D}$ & & \\
\hline 34 & $\begin{array}{c}\text { Ranchito San } \\
\text { Luís } \\
\end{array}$ & $\mathrm{E}$ & $\mathrm{X}$ & $\mathrm{X}$ & $X$ & 2 & $\mathrm{D}$ & & \\
\hline 38 & Banco de Tierra & $\mathrm{E}$ & $\mathrm{X}$ & $\mathrm{X}$ & $\mathrm{X}$ & 2 & $\mathrm{D}$ & & \\
\hline 39 & $\begin{array}{c}\text { Potrero de } \\
\text { Ubala }\end{array}$ & $\mathrm{E}$ & $X$ & $\mathrm{X}$ & $X$ & 2 & $\mathrm{D}$ & & \\
\hline
\end{tabular}




\begin{tabular}{|c|c|c|c|c|c|c|c|c|c|}
\hline 88 & $\begin{array}{l}\text { San Antonio } \\
\text { La Meseta }\end{array}$ & $\mathrm{E}$ & $\mathrm{X}$ & $\mathrm{X}$ & $\mathrm{X}$ & 5 & $\mathrm{D}$ & & \\
\hline 106 & Suchila & $E$ & $X$ & $X$ & $X$ & 5 & D & & \\
\hline 112 & Chicutan & $\mathrm{S}$ & $X$ & $\mathrm{X}$ & $X$ & 6 & $\mathrm{D}$ & & \\
\hline 118 & El Mostacillo & $\mathrm{E}$ & $\mathrm{X}$ & $\mathrm{X}$ & $\mathrm{X}$ & 6 & I & & \\
\hline 120 & Ashin norte & $\mathrm{E}$ & $X$ & $\mathrm{X}$ & $X$ & 6 & I & & \\
\hline 125 & Chamumún Sur & $\mathrm{E}$ & $\mathrm{X}$ & $\mathrm{X}$ & $\mathrm{X}$ & 6 & $\mathrm{D}$ & & \\
\hline 3 & Las Conchitas & $\mathrm{E}$ & & $X$ & & 1 & I & & \\
\hline 101 & $\begin{array}{l}\text { Loma de San } \\
\text { Jacinto }\end{array}$ & $\mathrm{S}$ & & $\mathrm{X}$ & & 5 & $\mathrm{D}$ & & \\
\hline 16 & Las Abejas & $\mathrm{S}$ & & $\mathrm{X}$ & $\mathrm{X}$ & 1 & $\mathrm{D}$ & & \\
\hline 20 & $\begin{array}{c}\text { Loma Potrero } \\
\text { Sivaca }\end{array}$ & $\mathrm{S}$ & & $\mathrm{X}$ & $\mathrm{X}$ & 1 & $\mathrm{D}$ & & \\
\hline 26 & Chenmax & $\mathrm{S}$ & & $X$ & $X$ & 1 & D & & \\
\hline 27 & Las Navajas & $\mathrm{E}$ & & $\mathrm{X}$ & $\mathrm{X}$ & 1 & $\mathrm{D}$ & & \\
\hline 31 & Ubala & $\mathrm{E}$ & & $X$ & $\mathrm{X}$ & 2 & $\mathrm{D}$ & & \\
\hline 42 & Alacté & $\mathrm{E}$ & & $\mathrm{X}$ & $\mathrm{X}$ & 3 & $\mathrm{D}$ & $\mathrm{X}$ & \\
\hline 44 & Mumuntic & $\mathrm{S}$ & & $X$ & $X$ & 3 & $\mathrm{D}$ & & \\
\hline 57 & $\begin{array}{l}\text { San Vicente } \\
\text { La Palma }\end{array}$ & $\mathrm{S}$ & & $\mathrm{X}$ & $X$ & 3 & I & & \\
\hline 60 & Campomtic & $\mathrm{S}$ & & $\mathrm{X}$ & $\mathrm{X}$ & 3 & $\mathrm{I}$ & & $\mathrm{X}$ \\
\hline 92 & $\begin{array}{c}\text { Rancho Antonio } \\
\text { Alvorez }\end{array}$ & $\mathrm{E}$ & & $\mathrm{X}$ & $\mathrm{X}$ & 5 & $\mathrm{D}$ & $\mathrm{X}$ & \\
\hline 93 & $\begin{array}{c}\text { El } \\
\text { Desembocadero }\end{array}$ & $\mathrm{S}$ & & $\mathrm{X}$ & $\mathrm{X}$ & 5 & I & & \\
\hline 98 & San Jacinto & $\mathrm{E}$ & & $\mathrm{X}$ & $\mathrm{X}$ & 5 & $\mathrm{D}$ & $X$ & \\
\hline 100 & $\begin{array}{l}\text { Potrero de San } \\
\text { Jacinto }\end{array}$ & $\mathrm{S}$ & & $\mathrm{X}$ & $\mathrm{X}$ & 5 & $\mathrm{D}$ & & \\
\hline 146 & Flor de Caña & $\mathrm{S}$ & & $\mathrm{X}$ & $\mathrm{X}$ & & $\mathrm{I}$ & & \\
\hline 115 & Cololté B & $\mathrm{S}$ & & & $\mathrm{X}$ & 5 & $\mathrm{D}$ & & \\
\hline 41 & Tzajalchib & $\mathrm{E}$ & & & $\mathrm{X}$ & 2 & $\mathrm{D}$ & $X$ & \\
\hline 85 & Petultón & & $\mathrm{X} ?$ & & & & $\mathrm{D}$ & $\mathrm{X}$ & \\
\hline 95 & Las Angustias & & $\mathrm{X}$ ? & & & & $\mathrm{D}$ & $\mathrm{X}$ & \\
\hline 109 & San Ricardo & & $\mathrm{X}$ ? & & & & & $X$ & $\mathrm{X}$ \\
\hline 132 & Pamtela A & & $\mathrm{X}$ ? & & & & & & $\mathrm{X}$ \\
\hline 133 & Pamtela B & & $\mathrm{X} ?$ & & & & & & \\
\hline 135 & Chijilté & & $\mathrm{X} ?$ & & & & & & $\mathrm{X}$ \\
\hline
\end{tabular}

Desde un punto de vista global el número de sitios en el valle parecía reducido y queríamos verificar en qué proporción podrían habérsenos escapado los asentamientos menores. Debido a la presencia en 1979 y 1980 de campos donde el zacate alto y el ganado dificultaban la identificación de sitios menores o de plataformas bajas en la parte central del valle, el reconocimiento de esa zona (n. ${ }^{\circ} 3$ ) sólo proporcionó 9 asentamientos, representando un total de 26 estructuras. En 1993 se efectuó un reconocimiento de control de 18 km² 


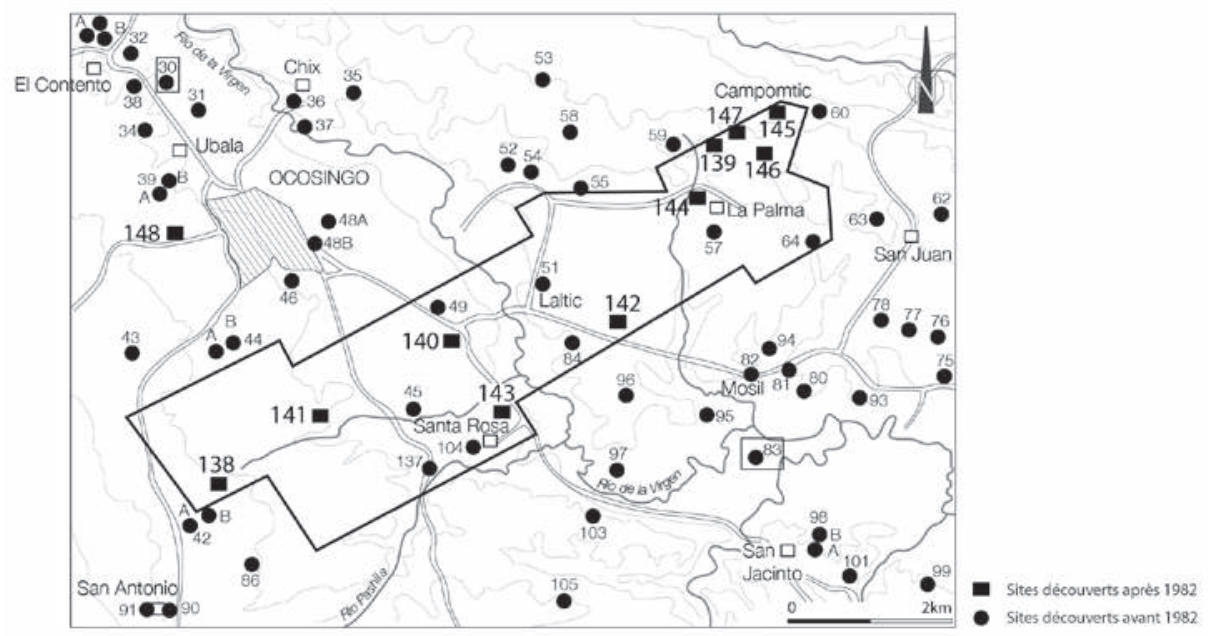

Fig. 7 - La zona de control de 1993, en la parte central del valle de Ocosingo (dib. Sylvie Éliés).

(Figura 7), que permitió ubicar siete nuevos asentamientos en esta zona, la mayoría de los cuales están compuestos de una sola estructura. Dos cuentan con tres plataformas y sólo el sitio de San Vicente Fracción proporcionó el material de superficie necesario para fecharlo en el Clásico Tardío. Se registraron también cuatro nuevos sitios fuera de la zona de reconocimiento, cerca de Campomtic en el valle norte, con un total de 13 estructuras. Al mismo tiempo, la última temporada permitió suprimir del registro el sitio de Mumuntic Alto (n. $\left.{ }^{\circ} 43\right)$, conformado por piedras calizas. Incluso con esos cambios el número de sitios y de plataformas sigue siendo reducido y no modifica drásticamente la escasez relativa. Una vez eliminados los casos dudosos y los ingenios, el total de asentamientos arqueológicos prehispánicos registrados y confirmados llega apenas a 111 (Figura 6) ${ }^{8}$.

\section{Evolución cronológica del asentamiento}

La Figura 6 sólo incluye los 82 sitios para los cuales se dispone de fechas seguras. Las publicaciones de Yadeun $(1992,1993)$ no hacen mención a investigaciones en el valle, ni al descubrimiento de otros sitios. Posiblemente algunos grupos menores de la periferia inmediata del sitio, considerados como sitios en 1981 (Tóniná Este alto y bajo, Rancho Cruz, El Sacramento, Miradero), formen parte ahora de los suburbios de Tonina (Figura 8). Su número

8. Por supuesto, los números que faltan en el Figura 6 (por ejemplo 1 o 4 ) corresponden a los sitios eliminados del registro. 


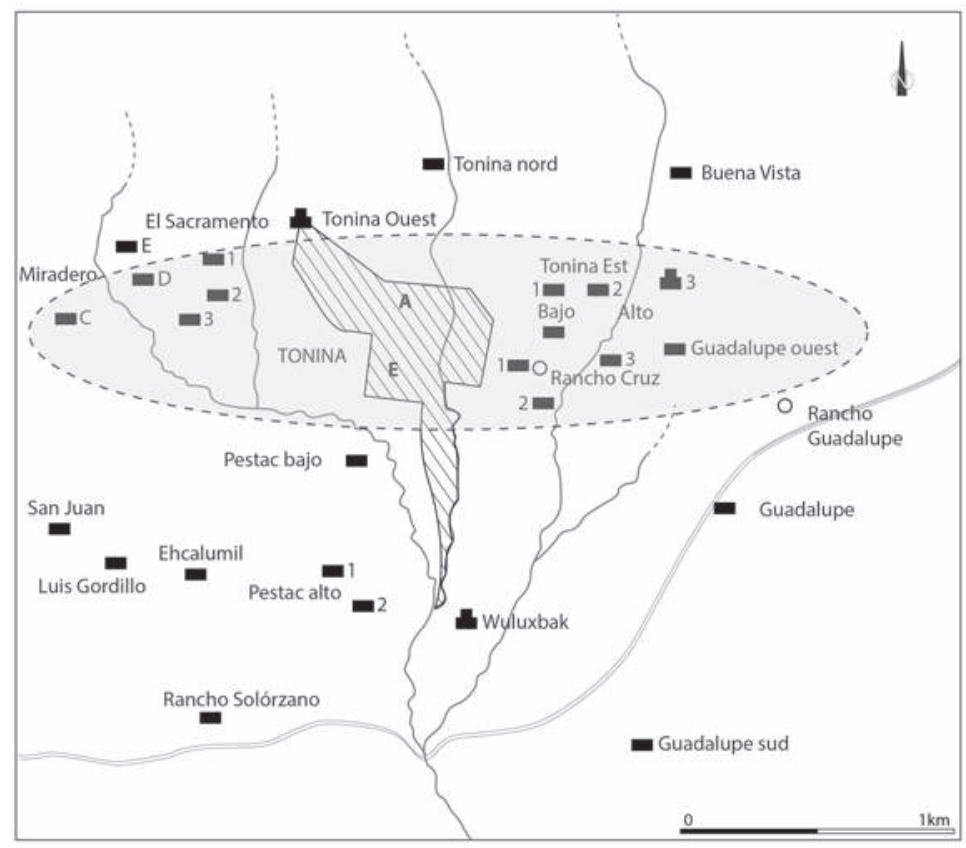

Fig. 8 - Los sitios periféricos de Tonina (dib. Sylvie Éliés).

resulta reducido y es poco probable que modifique significativamente el patrón de asentamiento tal como se presenta a continuación.

En los capítulos dedicados al estudio del patrón de asentamiento del valle de Ocosingo, Becquelin (Becquelin, Baudez [dir.] 1982; Becquelin, Taladoire [dir.] 1990) presentó una detallada descripción de los edificios y la tipología. No es necesario volver a esos aspectos. Más bien parece pertinente insistir aquí sobre el patrón global de la red de asentamiento y sus implicancias para la comprensión de la ocupación humana del valle.

Como se mencionó arriba, al momento de detallar la secuencia cronológica (Becquelin y Baudez 1979-1982) el concepto de Clásico Terminal todavía estaba mal definido. Queda ahora confirmada una ocupación continua, aunque de menor importancia, por lo menos hasta fines del siglo x (Taladoire 2016). En Tonina mismo, la estructura F5-2 en la segunda terraza de la Acrópolis atestigua actividades de construcción posteriores a la caída de la ciudad, o sea durante el Clásico Terminal, con la reubicación de monumentos anteriores (M. 122) (Becquelin y Taladoire 1990). Arnauld (1990) había notado ya, en el basurero del grupo F4-5 (en la primera terraza), la continuidad del material cerámico entre las fases Ixim y Chenek. Las excavaciones de Yadeun (com. pers. 2008) confirman en la parte oriental del sitio la presencia de actividades constructivas importantes de la propuesta fase Ixim/Chenek, con la edificación 
de un pequeño palacio. Es posible sugerir entonces, sin poder comprobarla todavía, la existencia de una fase de transición Ixim-Chenek (840-1000) del Clásico Terminal (Taladoire 2015, 2016), en concordancia con la cronología propuesta por Becquelin, Martin y Grube (2000) que presenta una secuencia dinástica continua hasta 910 . Debemos notar sin embargo que existe un lapso mal documentado en la secuencia, entre el reino de Uh Chapat, alrededor de 840 d.C. y el dirigente 10, con sólo dos monumentos fechados (M. 158 y M. 101). Ambas esculturas corresponderían entonces a la fase Ixim-Chenek del Clásico Terminal.

Disponemos de 82 sitios fechados (Tonina incluido), lo que corresponde a 115 ocupaciones. El complejo Wach del Preclásico Tardío cuenta con 8 ocupaciones, mientras el complejo May del Clásico Temprano está solamente representado por 4 (Figura 9). El complejo Ixim del Clásico Tardío (Figura 10), que corresponde al apogeo, es obviamente el mejor representado con 60 ocupaciones. Pero el Posclásico Temprano (complejo Chenek) alcanza 15 ocupaciones comprobadas, a las cuales se suma probablemente la mayoría de las 25 ocupaciones fechadas del Posclásico en general (Figura 11). En el marco de esta revisión cronológica debemos considerar que algunas ocupaciones de los complejos Ixim y Chenek se fecharían efectivamente en el Clásico Terminal, o sea en la propuesta fase Ixim/Chenek. Eso modificaría las proporciones relativas de ocupaciones para cada fase (Ixim, de transición Ixim/Chenek y Chenek) sin alterar la continuidad. El complejo Chib del Posclásico Tardío sólo está documentado en tres sitios.

De los sitios fechados en el valle, 58 cuentan con una sola ocupación conocida. En la ausencia de excavaciones extensivas resulta difícil confirmar que no hayan sido ocupados anteriormente. De los 33 sitios fechados por recolecciones de superficie, sólo en cuatro se identificaron dos o más ocupaciones distintas a partir del material. En cambio, en 22 de los 25 sitios excavados fue confirmada la presencia de ocupaciones sucesivas. Es muy probable entonces que, entre los sitios fechados sólo a partir de material de superficie, más de la mitad hayan sido ocupados en fases anteriores, lo que aumentaría el número de ocupaciones pero no modificaría drásticamente la repartición espacial. La presencia de una escultura en Campomtic, por ejemplo, sugiere una ocupación del Clásico Tardío que no ha sido evidenciada.

Este último aspecto resulta especialmente significativo porque permite esbozar la evolución del asentamiento en el valle. Como escribió Becquelin (Becquelin, Taladoire [dir.] 1990), en todas las fases la mayoría los sitios están ubicados sobre las lomas areniscas y/o en las vertientes, fuera de las zonas bajas inundables. Se trata de una adaptación natural, que además presenta la ventaja de dejar disponibles las mejores tierras agrícolas. La única excepción corresponde a la fase Wach del Preclásico, donde los sitios de Mosil C y Tonina Norte se ubican en las planicies, cerca de los ríos. Pero, en la misma fase, Tonina, Chix 
El territorio de Tonina, Chiapas

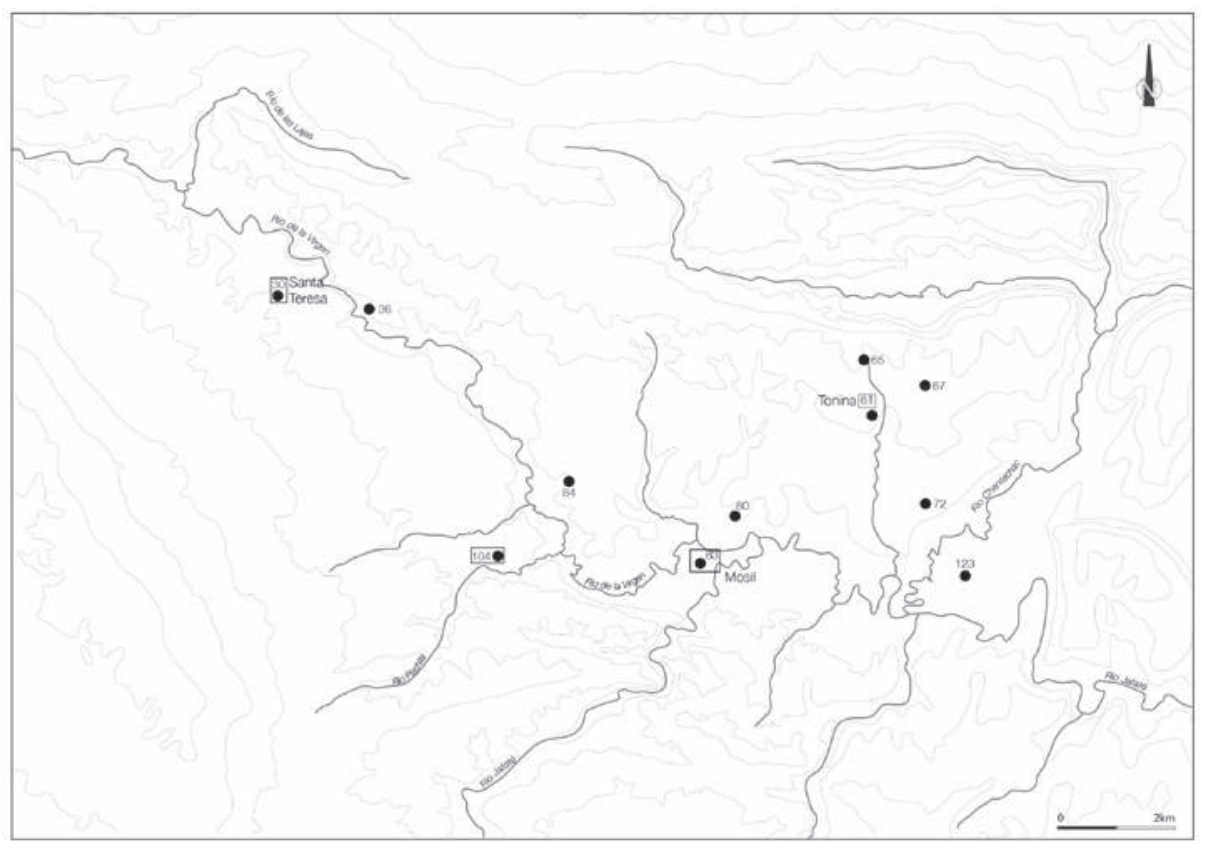

Fig. 9 - Repartición de la ocupación durante las fases Wach y May (dib. Sylvie Éliés).

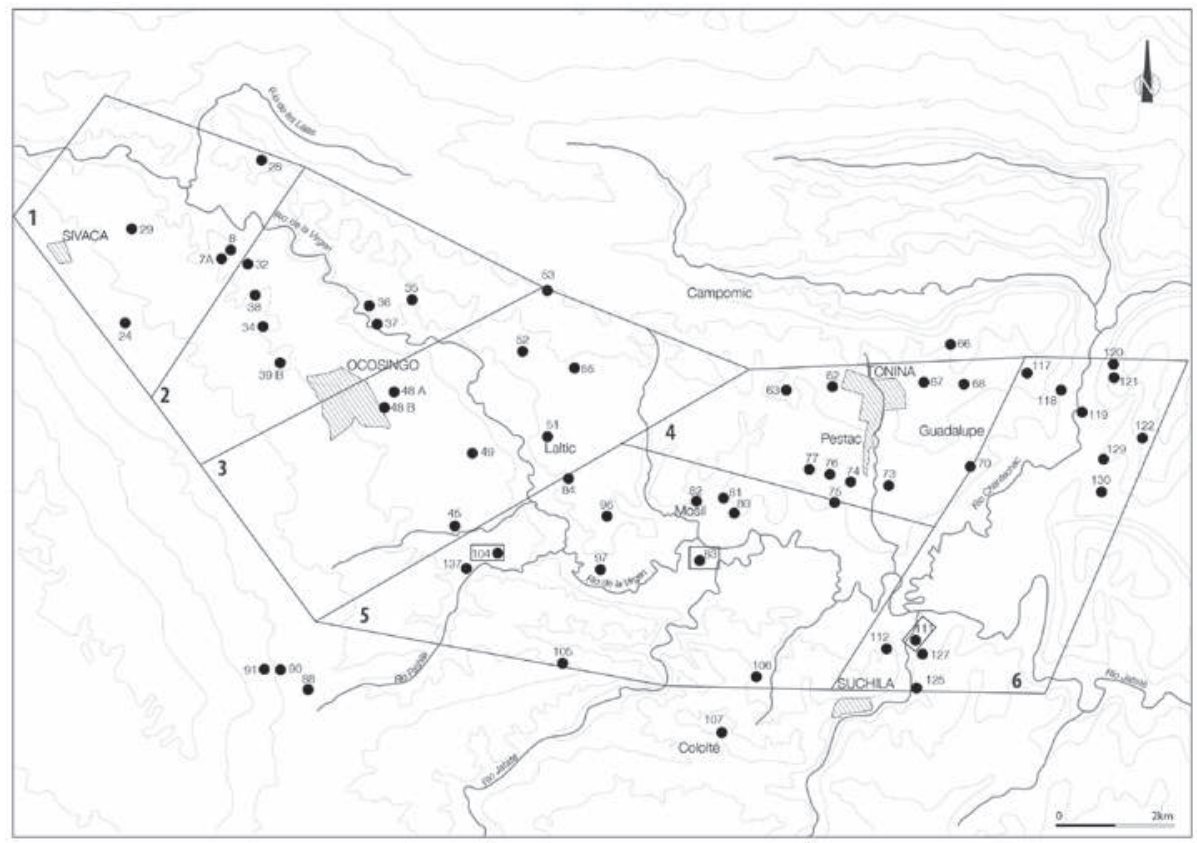

Fig. 10 - Repartición de los sitios durante la fase Ixim (dib. Sylvie Éliés). 


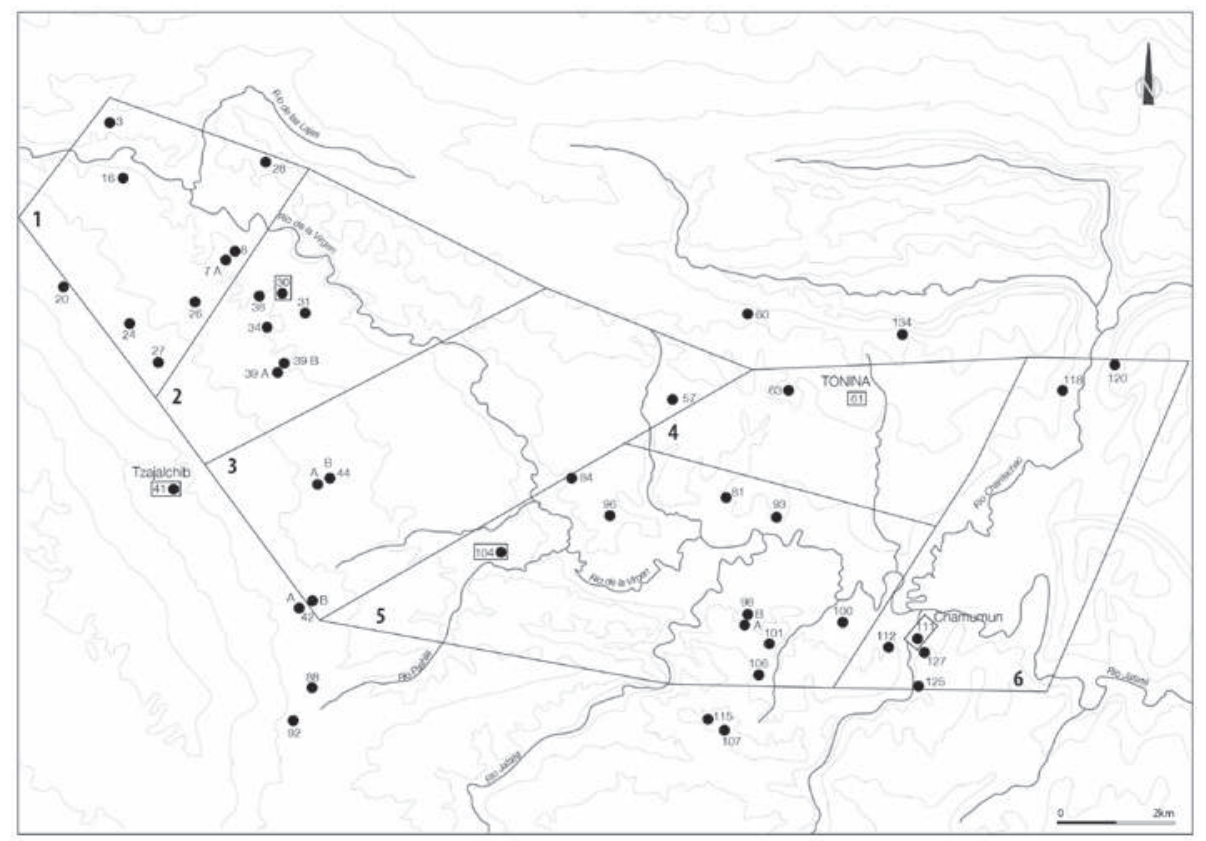

Fig. 11 - Repartición de la ocupación durante las fases Chenek y Chib (dib. Sylvie Éliés).

y Laltic se localizan en las faldas de las lomas. En la fase May, si bien Santa Teresa ocupa una loma de la parte baja del valle, su importancia no se puede equiparar con Tonina, donde las excavaciones recientes evidenciaron actividades del Clásico Temprano (Yadeun 1992, 1993). A partir de la fase Ixim, el valle se encuentra totalmente ocupado, aun cuando se registra una preferencia por la ribera izquierda del Jataté.

Registramos una evolución marcada en las fases Chenek y Chib (Figura 11). Aun cuando 10 sitios de la ribera norte siguen ocupados en Ixim/Chenek o Chenek, entre ellos Tonina, los centros mayores, Chamumún, así como la mayoría de los menores (Miradero, Cololté...), se ubican en la ribera derecha del Jataté. Si incluimos todos los sitios posclásicos, llegamos a 26 sitios, en comparación con los 10 de la margen izquierda9 ${ }^{9}$. Resulta más llamativo aún el asentamiento de la fase Chib (Tzajalchib y Cololté), ubicado en su totalidad en la parte alta meridional del valle. Este fenómeno refleja un cambio importante del centro de gravedad de la ocupación humana hacia el suroeste, rumbo a los Altos de Chiapas. Es difícil afirmar si esta evolución se justifica por razones

9. Este número podría aumentar si algunos de los asentamientos azucareros del siglo pasado, todos ubicados en la margen derecha del Jataté, recubren ocupaciones prehispánicas. 
económicas (el acceso a las tierras bajas del valle), políticas (la emergencia de un poder local en Chamumún luego de la matanza de la dinastía de Tonina), estratégicas (el Jataté formaría una barrera protectora) o bien por los lazos de los habitantes del valle con los Altos de Chiapas, de donde proviene la cerámica de la fase Chenek (Culbert 1965; Paris, Taladoire y Lee 2013, 2015).

\section{Asentamiento y demografía}

Del total de 111 sitios (Figura 6), 13 poseen monumentos, además de Tonina. Dos (Tonina Este y Oeste) forman parte de la periferia inmediata del sitio y podrían descontarse de la lista como suburbios de la ciudad (Figura 8). Puede retirarse también de la lista Mosil C, cuyos discos podrían fecharse del Preclásico. Cololté A se ubica en una loma frente a Tonina, del otro lado del valle, y su localización podría justificarse por su papel ritual ${ }^{10}$. Quedamos entonces con 9 sitios menores con esculturas.

En términos arquitectónicos, 19 sitios cuentan con estructuras de carácter público. Deben descartarse los centros que florecieron en fases distintas al Clásico Tardío: Mosil C, Tonina Norte para el Preclásico, Santa Teresa en la fase May, San Jacinto y Alacté para el Posclásico temprano y Tzajalchib en la fase Chib. Chamumún, el centro mayor de la fase Chenek o Ixim/Chenek, estuvo ocupado anteriormente, sin que se pueda determinar su importancia a falta de excavaciones intensivas. Por tanto, resulta prudente no tomarlo en consideración. Quedan entonces 12 sitios secundarios de la fase Ixim que cuentan con estructuras cívicas. La presencia de juegos de pelota en Mosil B y Petultón es especialmente llamativa (Taladoire 1980), ya que no es frecuente encontrar canchas en sitios menores. Sólo tenemos algunos ejemplos similares en El Tambo (Arnauld et al. 2004) o Chawak But'o'ob (Walling et al. 2012). Tres de esos 12 sitios cuentan simultáneamente con edificios mayores y esculturas: Laltic, Luís Coelo y San Ricardo.

Existe entonces una categoría intermedia de asentamientos entre Tonina y los grupos residenciales sencillos, que incluye unos 20 sitios caracterizados por la presencia de monumentos, juegos de pelota y/o estructuras públicas que los arqueólogos asocian con la élite (Figura 12, ver página siguiente). Eso sugiere la existencia en el valle de linajes importantes relacionados con Tonina, aunque capaces de reivindicar su relativa autonomía. El asentamiento refleja la existencia de una estratificación social distinta del esquema usual, que contrasta los sitios satélites y una élite residente en el centro.

El tema de la evaluación demográfica bien podría parecer superfluo, pero resulta significativo. Becquelin y Michelet (1994) discutieron la problemática del

10. Se documentó un caso parecido en el valle de Copán. 


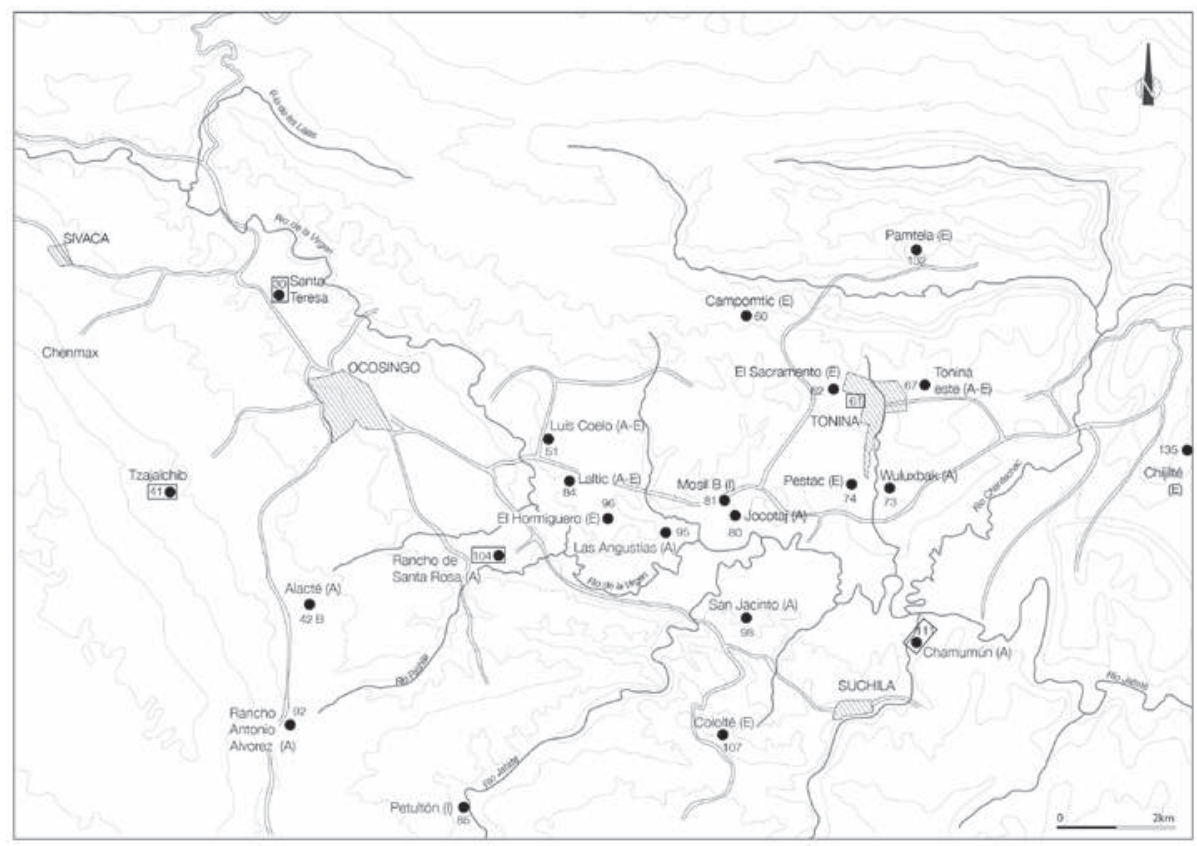

Fig. 12 - Ubicación de los sitios secundarios con monumentos (E) y/o arquitectura pública (A, I para juegos de pelota) en relación con la capital (dib. Sylvie Éliés).

cálculo de habitantes por casa o por plataforma. Si se utiliza usualmente el promedio de 5.6 personas por casa, las investigaciones de Breton (1979) en Bachajón y de Becquelin y Michelet en Xculoc (1994) proporcionan estimaciones diferentes: un promedio de 3 personas por casa en Bachajón y 3.4 personas en el Puuc. Para facilitar el análisis comparativo conservaremos provisionalmente la cifra de $5.6^{11}$. El análisis del asentamiento de Becquelin alcanza 260 plataformas residenciales para la fase Ixim, Tonina excluido (Becquelin, Taladoire [dir.] 1990). Habría existido en el valle una población total de 2356 habitantes, con unos 900 en Tonina y 1456 en el valle. Posteriormente, Becquelin (Becquelin, Michelet y Taladoire 1994) propone aumentar en un $42 \%$ el número de plataformas residenciales. Con esta corrección quedamos en 900-1000 para Tonina, acaso un poco más (si se añaden sitios periféricos, ver Figura 8), y 2060 en el valle; o sea un poco más de 3000, con una densidad de $29 \mathrm{~h} / \mathrm{km}^{2}$. En comparación con los datos del área rural de Copán, con 84 h/ $\mathrm{km}^{2}$ o Nohmul con 67 h/ $/ \mathrm{km}^{2}$ (Culbert y Rice 1990), el valle de Ocosingo parece entonces muy poco ocupado. Incluso los cálculos de Liendo Stuardo (2001) para los $40 \mathrm{~km}^{2}$ que rodean la ciudad de

11. Con cálculos basados en las estimaciones de Xculoc, la población del valle resultaría obviamente más reducida. 
Palenque en la fase Balunté corrigen la discrepancia: la densidad apenas llega a $25 \mathrm{~h} / \mathrm{km}^{2}$, cifra muy similar a la de Tonina, aun si la propia Palenque alcanzara unos 6000 u 8000 habitantes. Hasta incluyendo el valle norte (¿unos cientos de habitantes?), la base demográfica de Tonina parece definitivamente débil en comparación con la de otros sitios (Taladoire 2015). Pero esta escasez relativa, junto con la fertilidad natural del valle, sugiere que la población no necesitaba invertir mucho tiempo en las actividades de producción, y muchos habitantes estaban disponibles para otras actividades (Taladoire 2016).

Valía la pena mencionar esos datos porque caracterizan el valle y lo diferencian de otras entidades políticas vecinas. Pero resultaría aventurado relacionar directamente esa debilidad demográfica y la estructura socio-política que acabamos de mencionar con la política agresiva de Tonina. Al contrario: esa debilidad demográfica plantea el problema de la capacidad de Tonina para movilizar los efectivos suficientes para sus expediciones militares.

\section{Las implicaciones de la cerámica}

No se trata aquí de resumir los resultados del estudio de la cerámica, tal como se presentaron en los volúmenes publicados (Becquelin, Baudez [dir.] 1979; Becquelin, Taladoire [dir.] 1990), sino de extraer las conclusiones pertinentes para nuestro propósito.

La ocupación conocida del valle de Ocosingo empieza con el Complejo Wach, fechado por Becquelin de 0-300 d.C. Se caracteriza por la presencia de tipos de la esfera cerámica Chicanel que llega hasta Chiapas, con el grupo Sierra y material de tipo Usulután. Asimismo, el Complejo May (300-550 d.C.) pertenece claramente en la esfera cerámica Tzakol, con sus tipos característicos, Dos Arroyos Naranjo Policromo, Ixcanrio Naranjo Policromo.

El Complejo Ixim (600-910, según Becquelin, o 600-840 según la revisión propuesta en este trabajo) sigue la misma tradición, y sus relaciones con la esfera Tepeu se confirman principalmente con Pomoná (García Moll 2005) ${ }^{12}$, por más que se noten diferencias mínimas que podrían explicarse por su ubicación periférica (Taladoire 2016). Rands (1967) subraya que la cerámica de Tonina se diferencia mucho de la de Palenque y define la frontera entre ambas zonas a medio camino, en el río Tulija: así, por ejemplo, la proporción de tipos policromos es menos importante en Tonina que en otros sitios. Se nota en especial la baja frecuencia del grupo Gris Fino (Chablekal). Importa mencionar aquí que los últimos estudios cerámicos (Rands, Bishop y Sabloff 1982; Demarest, Rice P. y Rice D. S. 2004; Forsyth 2005; Forné et al. 2010) ubican definitivamente el grupo Gris Fino (Chablekal) en el Clásico Tardío, antes del Clásico Terminal.

12. García Moll compara el material de Pomoná con los tipos Ashin o Pamtela de la fase Ixim de Tonina. 
Durante la posible fase de transición Ixim-Chenek se nota la presencia, aunque escasa, de cerámica Naranja Fina y una cantidad difícil de estimar de recipientes plomizos (Tohil). En esta fase también se registran semejanzas con los tipos San Gregorio Coarse, Huistan hard, Ucutsin Brown on buff de los Altos de Chiapas (Culbert 1965; Paris, Taladoire y Lee 2015, p. 107). Becquelin había notado ya semejanzas estilísticas entre algunos recipientes Laltic Naranjo policromo e Ixtapa Fino de Ecatepec (Becquelin y Baudez 1979).

La situación cambia definitivamente con el Complejo Chenek (ca. 10001250), que no presenta rasgo alguno de las tierras bajas. Sus tipos característicos, Huistan Duro, San Gregorio burdo y Ucutsin, proceden de los Altos (Culbert 1965; Paris, Taladoire y Lee 2013, 2015). Hay todavía algunos anaranjados finos (Silho) y algo de Tohil Plomizo, con relaciones esporádicas con regiones lejanas. El Complejo Chib (1250-1528) es comparable con el Complejo Lum de los Altos de Chiapas (Culbert 1965), con los mismos tipos Huistan Hard y San Gregorio Burdo.

Para sintetizar, el material cerámico del valle de Ocosingo durante las fases Wach, May e Ixim se ubica en la esfera de las tierras bajas mayas, aun si se registran diferencias que podrían proceder de su ubicación periférica. Como lo había notado Arnauld (1990), basándose en sus análisis estratigráficos y estadísticos, la situación cambia paulatinamente, con cierto grado de continuidad, en la fase de transición Ixim/Chenek y al principio del Posclásico, con su integración en las tradiciones de los Altos de Chiapas. Falta mucho todavía para interpretar esa evolución y se han adelantado varias hipótesis al respecto. Pescador (2006) propone la llegada de nuevos habitantes procedentes del norte de Chiapas. Pero los cambios progresivos en la cerámica se asemejan con la permanencia de varios asentamientos y la ocupación relativamente densa del valle, lo que sugiere una continuidad. Además, a pesar de la destrucción violenta de Tonina, se evidencian en la ciudad actividades constructivas y tentativas de restaurar ciertos monumentos (M. 122), lo que implica que parte de la población local pertenecía a la élite anterior que trataba de perpetuar sus tradiciones.

Otra hipótesis sería entonces que parte de los habitantes del valle logró sobrevivir a la matanza. Los cambios en la cerámica se justificarían por la ruptura definitiva de las relaciones con las tierras bajas, donde la mayoría de las ciudades rivales (Palenque, Yaxchilán o Piedras Negras) ya habían dejado de existir. Los sobrevivientes de Tonina se habrían vuelto entonces hacia sus vecinos del suroeste, en los Altos, y con los nuevos intercambios se produjo la llegada de nuevos habitantes con tradiciones propias (Paris, Taladoire y Lee 2013, 2015).

Ayala $(1997,2002)$ adelantó la hipótesis de un origen tzeltal de la población del territorio de Tonina, e incluso del valle mismo. Wichman y Lacadena (2005) subrayan también la presencia de elementos tzeltales en las inscripciones. Eso sugiere un parentesco étnico entre los habitantes del valle y los de los Altos de Chiapas, que facilitaría su integración (Taladoire 2015). Esta hipótesis resulta 
frágil pero es compatible con el cambio mencionado del centro de gravedad del asentamiento, a principios de la fase Ixim/Chenek, y con las pocas diferencias registradas entre las ocupaciones de las fases Ixim final y Chenek. La presencia de linajes nobles ubicados en los sitios menores con monumentos y edificios cívicos en este estrato intermedio que hemos identificado sería un factor de continuidad, sin que esos linajes fueran capaces de restaurar el prestigio anterior de Tonina. El examen de los datos estilísticos y epigráficos proporciona argumentos suplementarios.

\section{El estilo escultórico}

Desde las primeras exploraciones, el estilo escultórico de Tonina ha llamado la atención. Blom y LaFarge (1926-1927) registraron unos 30 monumentos, y a lo largo de las visitas, el total de monumentos conocidos ha alcanzado alrededor de 50, incluyendo algunas piezas de sitios del valle como la estela de Pestac (Blom 1935). Al finalizar nuestras excavaciones el registro de Mathews (1983) y de Graham y Mathews $(1996,1999)$ llegaba a 141 monumentos, 5 misceláneas, la estela de Pestac, 3 piezas de colecciones privadas y más de 115 fragmentos. Abarcando la mayoría de las piezas encontradas por el proyecto de Yadeun, el estudio complementario de Graham (2006) llevó ese total a 176 monumentos y 6 fragmentos nuevos, sin contar una miscelánea más. Nuevos monumentos siguen apareciendo en las últimas excavaciones (Yadeun, com. pers. 2008; Stuart 2011). Pueden añadirse en la lista dos esculturas sin procedencia (Mayer 2007a y b) que, si son del estilo escultórico de Tonina, podrían proceder de otros sitios (Guaquitepec o Santo Ton, por ejemplo).

Para evaluar la importancia de este corpus basta recordar que, según Mathews (1997), sitios mayores como Dos Pilas, Seibal o Yaxchilán sólo cuentan con respectivamente 34, 29 y 162 monumentos con inscripciones. Además, a partir del corpus de Tonina, Graham (2006) identificó una nueva categoría de monumento, el pedestal, que figura entre los rasgos estilísticos que caracterizan a Tonina junto con las estatuas, la omnipresencia de representaciones de cautivos y el uso de la piedra arenisca. Si Tonina comparte algunos rasgos con otros sitios (las representaciones de cautivos con espiga con Tenam Puente o los motivos en estuco con Palenque), no cabe duda de que se trata de un verdadero estilo regional que permite incluir en la esfera estilística de Tonina a sitios como Quexil/San Ricardo, Santo Ton y Guaquitepec (Mayer 1984), para limitarnos a los principales. Todavía es imposible determinar si los monumentos de Santa Elena Poco Uinic se parecen a los de Tonina. La nota de Mathews (Wayeb s.f.) indica la presencia, en la inscripción de la estela 3, de un dirigente local, Yax B'alam, (nacido en 766 d.C. [?], en el poder entre 782-790). De acuerdo con Mathews, es más prudente considerar que Santa Elena Poco Uinic sea la capital de una pequeña entidad política independiente, más bien relacionada 
con Chinkultic y Tenam Puente. Si tal fuera el caso Santa Elena Poco Uinic podría ubicarse entre los vecinos y tal vez los adversarios de Tonina, limitando así su territorio hacia el sureste (Figura 2).

Resulta significativo insistir sobre la repartición, en la periferia de Tonina y en su territorio, de los sitios secundarios con inscripciones. Como muchas ciudades mayas, la entidad política de Tonina posee centros satélites. Los de Yaxchilán se encuentran en La Pasadita, El Chicozapote y Ojos de Agua, los de Piedras Negras en El Cayo y La Mar. Pero, considerando las distancias entre las capitales y los centros satélites (Figura 13), se nota de inmediato una diferencia marcada. Alrededor de Palenque, Pomoná, Piedras Negras, Yaxchilán o Bonampak, la distancia promedio entre la capital y los centros secundarios es de $20 \mathrm{~km}$. Puede alcanzar $48 \mathrm{~km}$ entre Palenque y Tila, y hasta $60 \mathrm{~km}$ entre Palenque y Tortuguero, pero casi nunca cae a menos de $10 \mathrm{~km}$, salvo Panhalé que se encuentra a $8 \mathrm{~km}$ de Pomoná y Kuna a $6 \mathrm{~km}$ de Bonampak. Globalmente, los centros secundarios se ubican en los confines del territorio de cada entidad (Figura 2). En la entidad de Tonina, por el contrario, Guaquitepec se encuentra a una distancia de $35 \mathrm{~km}$ y Santo Ton a $14 \mathrm{~km}$. Cabe notar que la región de Guaquitepec corresponde posiblemente a otra entidad, Sib'ikte, estrechamente ligada con Tonina pero tal vez autónoma (Taladoire 2015). Todos los demás sitios se ubican en el valle mismo, a menos de $7 \mathrm{~km}$, o sea mucho más cerca del sitio capital. Se nota un patrón de concentración marcado alrededor de Tonina sin borrar toda descentralización, ya que no había linajes importantes radicados en el mero centro (Figura 12).

\section{Los datos epigráficos}

Los datos epigráficos proporcionan elementos que aclaran hasta cierto punto este patrón de dispersión en la periferia inmediata del sitio. No es pertinente volver a presentar aquí la secuencia dinástica de Tonina, que ha sido el enfoque de estudios recientes (Martin y Grube 2000; Mathews 2001). Sólo mencionaremos que existen lagunas importantes, que posiblemente se clarifiquen con nuevos descubrimientos: así, el número de dirigentes está todavía mal definido. Martin y Grube (2000, p. 178) escriben por ejemplo: "Ruler 1, in a sequence of ten Tonina kings compiled by Peter Mathews", mientras que su propia lista incluye 12 dirigentes, cuatro de ellos sin nombre y uno desconocido. Ayala (2002) plantea además la hipótesis del papel original de las mujeres en la sucesión dinástica y hasta adelanta la posibilidad de un modo distinto de designación de los dirigentes. Lo que se presenta a continuación, entonces, no puede ser más que un esbozo.

La primera mención del glifo emblema de Tonina se encuentra en un trono de Chinikihá, un centro menor de Pomoná a $72 \mathrm{~km}$ al norte de Tonina: en 573, un personaje de Tonina habría sido capturado (Martin y Grube 2000). Eso implica 


\begin{tabular}{|c|c|c|}
\hline Sitios & Tipos de monumentos & Distancia estimada \\
\hline \multicolumn{3}{|c|}{ Palenque } \\
\hline Chuctiepa & Estela, altar & $20 \mathrm{~km}$ \\
\hline Miraflores & Fragmento & $16 \mathrm{~km}$ \\
\hline Tortuguero & Lapidas & $60 \mathrm{~km}$ \\
\hline El Retiro & $?$ & $27 \mathrm{~km}$ \\
\hline Xupa & Fragmento & $11 \mathrm{~km}$ \\
\hline La Preciosa & $?$ & $28 \mathrm{~km}$ \\
\hline Tila & Estela & $48 \mathrm{~km}$ \\
\hline Chinikihá & Trono & $37 \mathrm{~km}$ \\
\hline La Cascada & Estela & $22 \mathrm{~km}$ \\
\hline \multicolumn{3}{|c|}{ Pomoná } \\
\hline Panhalé & Estela & $8 \mathrm{~km}$ \\
\hline \multicolumn{3}{|c|}{ Piedras Negras } \\
\hline El Cayo & Paneles & $12 \mathrm{~km}$ \\
\hline La Mar & Estelas & $16 \mathrm{~km}$ \\
\hline \multicolumn{3}{|c|}{ Yaxchilán } \\
\hline La Pasadita & Dinteles & $15 \mathrm{~km}$ \\
\hline El Chicozapote & Dinteles & $20 \mathrm{~km}$ \\
\hline Ojos de Agua & ? & $20 \mathrm{~km}$ \\
\hline \multicolumn{3}{|c|}{ Lacanhá-Bonampak } \\
\hline Kuna-Lacanhá & Dintel & $6 \mathrm{~km}$ \\
\hline Nuevo Jalisco & Paneles & $?$ \\
\hline Tzendales & Estela & $42 \mathrm{~km}$ \\
\hline Oxlahuntun & Estela? & $16 \mathrm{~km}$ \\
\hline \multicolumn{3}{|c|}{ Santa Elena Poco Uinic } \\
\hline El Amparo & ? & $4 \mathrm{~km}$ \\
\hline \multicolumn{3}{|c|}{ Chinkultic } \\
\hline La Esperanza & Disco & $10 \mathrm{~km}$ \\
\hline Sacchana & Estela & $7 \mathrm{~km}$ \\
\hline \multicolumn{3}{|c|}{ Tonina } \\
\hline Guaquitepec & Estela y altar & $35 \mathrm{~km}$ \\
\hline Santo Ton & Estelas y otros & $14 \mathrm{~km}$ \\
\hline Miradero & Panel & $1 \mathrm{~km}$ \\
\hline Pestac & Estela & $1 \mathrm{~km}$ \\
\hline Quexil & Pedestal & $7 \mathrm{~km}$ \\
\hline Chijilté & Disco con glifos & $6 \mathrm{~km}$ \\
\hline Cololté A & Estatua & $6 \mathrm{~km}$ \\
\hline Tonina Este Bajo & Estatua (M. 126) & $0.5 \mathrm{~km}$ \\
\hline
\end{tabular}

Fig. 13 - Repartición de los monumentos con inscripciones de los sitios secundarios de las diferentes entidades políticas de las tierras bajas occidentales. 
que Tonina ya tenía suficiente importancia para ser mencionada aun cuando no se conoce nada de su historia en el Clásico Temprano. En 633, el dirigente K'inich Hix Chapaht (o K'inich B'olon Chapaht) instala señores menores en unos oficios que Martin y Grube (2000) transcriben como aj k'uhuun y nuun (M. 154). Ellos parecen tener un papel importante, pues se mencionan en otras inscripciones, y siguieron asociados con la dinastía local, puesto que reaparecen en monumentos consagrados a dirigentes posteriores.

El dirigente siguiente, cuyo nombre sigue siendo desconocido, pudo haber sido capturado durante una guerra con Palenque. Una inscripción del templo XVII de Palenque menciona la conquista de Tonina por los palencanos en 687 y la posible muerte del dirigente. Este último es reemplazado en 688 por K'inich B'aaknal Chaak, con el apoyo de los aj k'uhuun y nuun de sus predecesores. Este soberano inicia una política agresiva y conduce una primera expedición hacia el noroeste, luego una segunda hacia la entidad mal conocida de Anay Te' (¿tributaria de Yaxchilán o, según Mathews, de Palenque?) en el área del Usumacinta, y una tercera contra La Mar, tributaria de Piedras Negras. Varias representaciones de cautivos sugieren que Tonina estuvo en conflicto permanente con otras ciudades, siendo Palenque a unos $65 \mathrm{~km}$ al norte su enemigo principal. Una nueva guerra termina con la captura de Sak B'alam de Palenque. Un monumento descubierto por Yadeun documenta asimismo la captura de Buk' (mencionado en el M. 145), en 692 (Stuart 2011). Finalmente, en 711, los guerreros de Tonina se apoderan de Palenque y de su dirigente K'an Joy Chitam (M. 122).

Según algunas lecturas del M. 153, en 716 Tonina habría estado bajo el control de Calakmul, pero en 717, el siguiente dirigente, de nombre desconocido, retoma la política agresiva de su predecesor y vence en 721 a un jefe secundario de Calakmul, originario del Grupo Chik Nab (Carrasco y Bojalil 2005). Dada la distancia entre Calakmul y Tonina, un conflicto directo resulta imposible. El sitio vencido podría entonces ser aliado de Calakmul, pero ¿dónde se ubicaría? Podría provenir de Pomoy, al noroeste, cerca de Tortuguero. Efectivamente, la entidad política de Palenque está aliada con Tikal, hostil a Calakmul. Lo mismo puede suponerse de sus sitios satélites como Tortuguero. Sea lo que fuere, notamos que tanto Palenque como Tonina comparten la misma hostilidad hacia Calakmul, sin por lo tanto refrenar su animosidad mutua. Eso sugiere que Tonina no estaba involucrada en las esferas políticas de Tikal y Calakmul, persiguiendo su política propia, relativamente independiente.

Entre 736 y 775, las inscripciones en Tonina son muy escasas. Mathews (2001) supone que los monumentos relativos a este intermedio están ubicados en otra parte del sitio, donde todavía restan por descubrirse. También puede contemplarse la hipótesis de una crisis consecutiva a una derrota. En 786 Tonina ha recuperado un poder suficiente para volver a hostigar, bajo el reino del dirigente $\mathrm{n}^{\circ} 8$, sus antiguos enemigos: Pomoy, el área del Usumacinta y posiblemente Sak T’zi (Plan de Ayutla), cuyo dirigente cayó prisionero (Martin 
y Grube 2000; Anaya Hernández, Guenter y Zender 2003). Los dirigentes siguientes siguen representando como sacrificados y cautivos sin que sea posible identificar las víctimas, pero después de 840 faltan datos epigráficos sobre la dinastía local luego de la muerte de Uh Chapat (Martin y Grube 2000) hasta el ascenso al poder del último dirigente $\left(\mathrm{n}^{\circ} 10\right)$, alrededor de $900^{13}$. En esta fecha los viejos enemigos de Tonina ya han caído. Es imposible determinar hacia dónde lanzaba sus ataques Tonina. Pero es posible que esas nuevas víctimas de la política de Tonina hayan podido contribuir al saqueo de la ciudad poco después de 909 d.C. y a la matanza de su élite.

Para resumir, resulta evidente que Tonina practicaba una política agresiva hacia sus vecinos, atacando indistintamente a los aliados de Tikal y de Calakmul y consiguiendo más victorias que derrotas. Sus principales enemigos eran Palenque y las diferentes entidades del valle del Usumacinta (Bonampak, Plan de Ayutla), pero sería fundamental investigar mejor la zona de Santa Elena Poco Uinic y las áreas fronterizas al este para complementar el panorama de sus adversarios potenciales. Recordemos que, si bien Tonina glorifica sistemáticamente sus victorias, con los glifos mencionando los emblemas de sus víctimas, raras veces se las menciona en otros sitios (Patrois 2000). Tal limitación reflejaría por supuesto las derrotas de sus enemigos, pero existen otras ocasiones en que las inscripciones refieren a otras ciudades: matrimonios, alianzas... Esta escasez de menciones podría reflejar un aislamiento relativo de Tonina. Confirma indirectamente la posición periférica del sitio y su ubicación imperfecta en la esfera cerámica de las tierras bajas.

El último aspecto que nos interesa aquí en relación con los datos epigráficos es la identificación de los oficios secundarios, aj k'uhuun y nuun, que, a pesar de su importancia en la historia del valle de Ocosingo, resultan difíciles de interpretar (Martin y Grube 2000; Lacadena 1996). Se diferencian obviamente de los sajales documentados para los sitios satélites de Palenque (Xupa, Miraflores), Pomoná (Panhalé) o Piedras Negras (El Cayo, La Mar) (Schele 1991; Biró 2012, p. 84-85). El título de sajal no aparece en los textos de Tonina. ¿Podría relacionarse esa diferencia con la ubicación de los sitios satélites en la periferia inmediata de Tonina? Debemos contemplar esa posibilidad sin tener los datos suficientes para comprobarla. De todos modos, los datos epigráficos confirman la presencia en el valle de una nobleza estrechamente asociada con la dinastía y distinta de los sajales de las otras entidades políticas. Si efectivamente los dirigentes de Tonina disponían de una nobleza intermedia, confiable y dispersa a lo largo del valle, eso les habría proporcionado un control más estrecho de su población, mientras los sajales de los satélites de otras entidades siempre podían pasar al control de otra ciudad o buscar más autonomía (Golden y Scherer 2013).

13. Ninguno de los últimos monumentos descubiertos por Yadeun permite documentar este lapso cronológico. 


\section{El territorio de Tonina}

Esta definición tentativa del territorio de Tonina y de su evolución, a través su patrón de asentamiento y los otros datos disponibles, no estaría completa si no considerásemos los estudios de la organización política de las tierras bajas. Basándose en la repartición conocida de los glifos emblemas, Marcus (1976) había propuesto una organización del territorio maya alrededor de varias capitales, incluyendo Tonina en la esfera de Palenque. Sabemos ahora que Tonina y Palenque eran entidades distintas y enemigas. Mathews (1997) propone una definición más empírica del territorio de cada ciudad, que correspondería con un área promedio de $2500 \mathrm{~km}^{2}$ (Figura 2). Por supuesto, el concepto de territorio corresponde más bien a relaciones sociopolíticas que a consideraciones prácticas de control de tierras ${ }^{14}$. Estos territorios no tienen fronteras sino límites imprecisos, incluso cuando, apoyándose en los datos cerámicos de Rands (1967), Mathews sugiere que el río Tulija, a medio camino entre Palenque y Tonina, casi tuvo un papel de frontera natural.

De acuerdo con su análisis, el territorio de Tonina, de una superficie superior a la de muchas otras entidades, habría limitado al norte con Palenque, al noreste con Pomoná, al este con Piedras Negras, Bonampak y Sak T’zi-Plan de Ayutla, mientras que al sur es incierto si el vecino más cercano era Chinkultic o Santa Elena Poco Uinic. Tratándose de esta última posibilidad, el territorio de Tonina habría sido ligeramente más reducido. La incógnita mayor es el límite suroccidental hacia los Altos de Chiapas. ¿Hasta dónde llegaba el dominio de Tonina en los Altos? ¿Cuáles eran sus relaciones con los sitios vecinos? Como ya dijimos, muchos elementos coinciden para atestiguar relaciones continuas entre los valles de Jovel y de Ocosingo, posiblemente facilitadas por una unidad lingüística (Ayala 1997, 2002; Paris, Taladoire y Lee 2013, 2015). ¿En qué medida la falta de enemigos en las tierras bajas condujo a los guerreros de Tonina a una política agresiva hacia los otros habitantes de esta zona? La caída violenta de Tonina no puede atribuirse a sus antiguos enemigos: ¿habrá sido debida a invasores de los Altos? La popularización del material cerámico de la fase Ixim/Chenek durante el Clásico Terminal y a principios del Posclásico podría explicarse por una irrupción de estos últimos. Pero quedaría por entender por qué los mismos invasores trataron, en Tonina como en otros sitios, de restaurar la importancia política del valle, retomando los antiguos símbolos de su agresividad a través de la presentación de cautivos como en la estructura F5-2. ¿Será que la nobleza intermedia del valle facilitó esa transición? Sólo un estudio intensivo de los sitios secundarios del valle de Ocosingo y de los sitios vecinos (Guaquitepec, Santa Elena Poco Uinic) podría brindar informaciones al respecto.

14. Para una discusión del concepto de territorio en el caso de Tonina, ver Taladoire 2015. 


\section{Tonina, una entidad original}

A manera de conclusión, pueden sintetizarse los datos y las hipótesis para tratar de entender lo que hace de Tonina y del valle de Ocosingo una entidad original en el ámbito de las tierras bajas mayas. El valle de Ocosingo ocupa una ubicación relativamente marginal en las orillas occidentales del área maya, en una posición intermedia entre las tierras bajas y los Altos de Chiapas. El valle forma una unidad geográfica bien definida, con verdaderos límites naturales, sin quedar no obstante aislado. El curso inferior del río Jataté proporciona un acceso fácil hacia el Usumacinta, mientras que su curso superior abre un camino hacia el valle de Chilón y, más adelante, hacia las planicies de Tabasco y la zona de Palenque (Taladoire 2016). La presencia de las tierras altas calizas en la vertiente meridional del valle facilita a su vez un acceso directo hacia los Altos.

Sin volver a insistir sobre las especificidades de Tonina, llaman la atención varios aspectos del patrón de asentamiento del valle. No se puede comparar el tamaño de Tonina con sus sitios tributarios, pero se nota la existencia de una categoría intermedia de asentamientos entre la capital regional y los grupos residenciales sencillos. Esta categoría intermedia, que incluye unos 20 sitios, se caracteriza por la presencia de rasgos que los arqueólogos asocian generalmente con la élite, o sea monumentos, juegos de pelota y/o estructuras públicas. Esto sugiere la existencia de linajes nobles asociados con la dinastía de Tonina en el valle de Ocosingo, pero residiendo fuera del centro (Figura 12). Es importante insistir sobre la cercanía entre esos asentamientos y Tonina, a diferencia de lo que ocurre en otras entidades. El uso de títulos poco comunes, como aj k'uhuun y nuun (Martin y Grube 2000; Lacadena 1996; Biró 2012, p. 84-85), tal vez se relacione entonces con ese patrón inusual, sobre todo si se considera que tales dignatarios desempeñaron un papel de larga duración junto a la dinastía.

El estudio del material cerámico del valle de Ocosingo durante las fases Wach, May e Ixim estableció la pertenencia de Tonina a la esfera de las tierras bajas mayas, incluso con diferencias. La situación cambia a fines del Clásico Tardío, o lo que proponemos considerar como Clásico Terminal (fase Ixim/ Chenek), con la integración progresiva del valle a la esfera Chenek de los Altos de Chiapas. Basándose en la antigua cronología, el cambio había sido previamente interpretado en términos de la llegada de inmigrantes procedentes del norte de Chiapas que habrían contribuido a la destrucción del sitio y a la matanza de su dinastía en 910. En el marco de la revisión cronológica que proponemos, Tonina habría sufrido hasta cierto punto de las consecuencias de la caída de muchas ciudades vecinas, sin por tanto cesar sus actividades luego de 840 d.C. Los cambios posteriores a 840 permiten mantener sus actividades a la ciudad, con nuevos edificios y nuevos monumentos, aunque obviamente de menor calidad. Además, a pesar de la destrucción violenta de Tonina en 910, existen evidencias de una continuidad cultural hasta el fin del Clásico Terminal, 
tanto en la Acrópolis como en otros sitios ${ }^{15}$. También se registró una evolución geográfica en la repartición de los asentamientos en el valle. Es difícil determinar si tales cambios se justifican por razones económicas, políticas, estratégicas o bien si se trata efectivamente de la llegada de invasores.

Hipotetizamos que parte de la población del valle logró sobrevivir, entre ella algunos linajes menores de las élites. El cambio en la cerámica puede explicarse entonces por la ruptura de los lazos con las tierras bajas, ya despobladas. Los sobrevivientes de Tonina se habrían vuelto hacia sus vecinos tzeltales del suroeste, facilitando la llegada de nuevos habitantes que traían con ellos sus propias tradiciones. Esta última hipótesis resulta compatible con el cambio del centro de gravedad del asentamiento y la introducción masiva de un nuevo complejo cerámico, pero que tenía antecedentes en una posible fase de transición Ixim/Chenek, en la escasa diferencia entre la cantidad de ocupaciones registradas de las fases Ixim y Chenek y en la continuidad cultural manifiesta en Tonina y unos sitios secundarios. La presencia en el valle de este estrato sociopolítico intermedio de pequeños linajes, que habrían sobrevivido a la matanza, pudo haber facilitado esa transición.

Sería esencial disponer en el futuro de más datos sobre Plan de Ayutla, investigar la zona de Santa Elena Poco Uinic y profundizar los estudios en curso sobre Chinkultic, Tenam Puente, el valle de Jovel y las otras entidades de los Altos de Chiapas, a fin de complementar el panorama de la definición del territorio de Tonina y de sus interacciones. *

* Manuscrit reçu en octobre 2015, accepté pour publication en mai 2017.

Agradecimientos - Quiero, por supuesto, agradecer a mis maestros Pierre Becquelin y Claude F. Baudez su invitación a participar en las diferentes temporadas de excavación realizadas en Tonina desde 1971 hasta 1981, lo que fue determinante para mí. Sin su apoyo permanente, sus críticas, su ayuda, nunca hubiera podido realizar esta investigación. Agradezco también a todos los miembros de las diferentes temporadas del proyecto Tonina por sus discusiones, sus comentarios o su presencia, pero debo mencionar especialmente a Charlotte Arnauld por sus sugerencias fructíferas sobre la existencia de un Clásico Terminal en este sitio, tema que ha sido objeto de numerosas controversias. En la revisión de nuestras antiguas hipótesis, Chloé Andrieu me ha abierto nuevos senderos: gracias. Los organizadores de la VI Mesa Redonda de Palenque me invitaron en 2008 a presentar una síntesis de nuestras investigaciones. Aunque el presente artículo poco tiene que ver con el texto original de mi presentación, me dieron entonces la oportunidad de aquella revisión: quisiera manifestar entonces mi reconocimiento, así como también a todos aquellos colegas que presenciaron el evento, por sus comentarios. Finalmente, estoy en deuda con los dos colegas que leyeron y criticaron la versión preliminar de este artículo, y especialmente con Arthur Demarest, y que me permitieron enriquecer el texto y corregir numerosos puntos discutibles.

15. Tambien posiblemente en la región de Guaquitepec. 


\section{Referencias citadas}

Álvarez A. Carlos

1993 "El patrón de asentamiento en Las Margaritas, Chiapas y su cronología tentativa", in Juan Pedro Laporte, Héctor L. Escobedo y Sandra Villagrán de Brady (eds), VI Simposio de investigaciones arqueológicas en Guatemala, 1992, Museo Nacional de Arqueología y Etnología, Guatemala, p. 462-473.

Anaya Hernández Armando, Stanley P. Guenter y Marc U. Zender

2003 "Sak T'zi, a classic maya center: a locational model based on GIS and epigraphy". Latin American Antiquity, 14 (2), p. 179-191.

Andrieu Chloé, Christophe Helmke, Harri Kettunen, Eric Taladoire y Robert Tamba

2011 "New maya paintings at Bolonkin, Chiapas", Mexicon, 33 (5), p. 7-10.

ARNAUld Marie Charlotte

1990 “Opération III: nouvelles fouilles dans le groupe F4", in Pierre Becquelin, Eric Taladoire (eds), Toniná, une cité maya du Chiapas (Mexique), t. 4, Centre d'études mexicaines et centramémericaines (Études mésoaméricaines, 6 [4]), México, p. 1505-1529.

Arnauld Marie Charlotte, Véronique Breuil-Martínez y Erick Ponciano Alvarado (eds)

2004 La Joyanca (La Libertad, Guatemala). Antigua ciudad maya del noroeste del Petén, CEMCA/Asociación Tikal/Centro de Investigaciones Regionales de Mesoamérica, Guatemala.

Ayala Falcón Maricela

1997 "Who were the people of Tonina?", in Martha Macri y Anabel Ford (eds), The Language of Maya Hieroglyphs, Precolumbian Art Research Institute, San Francisco, p. 69-75.

2002 "Lady K'awil, goddess o and Maya warfare", in Traci Ardren (ed.), Ancient Maya women, AltaMira (Gender and archaeology series, 3), Walnut Creek (CA).

BeCQuelin Pierre y Claude F. BAudez (eds)

1979 Tonina, une cité maya du Chiapas (Mexique), t. 1, Mission archéologique et ethnologique française au Méxique (Études mésoaméricaines, 6 [1]), México.

1982a Tonina, une cité maya du Chiapas (Mexique), t. 2, Mission archéologique et ethnologique française au Méxique (Études mésoaméricaines, 6 [2]), México.

1982b Tonina, une cité maya du Chiapas (Mexique), t. 3, Mission archéologique et ethnologique française au Méxique (Études mésoaméricaines, 6 [3]), México.

Becquelin Pierre y Dominique Michelet

1994 "Demografía en la zona Puuc: el recurso del método", Latin American Antiquity, 5 (4), p. 289-311.

Becquelin Pierre, Dominique Michelet y Eric Taladoire

1994 "Prospection de contrôle dans la vallée d'Ocosingo, Chiapas, Mexique", Journal de la Société des américanistes, 80, p. 185-190.

BeCquelin Pierre y Eric Taladoire (eds)

1990 Tonina, une cité maya du Chiapas (Mexique), t. 4, Centre d'études mexicaines et centramémericaines (Études mésoaméricaines, 6 [4]), México.

BIRÓ Peter

2012 "Politics in the Western Maya region (III). The Royal and the Non-Royal Elite", Estudios de cultura Maya, 40, p. 78-96. 
BLOM Frans

1935 “The Pestac Stela”, Maya Research, 2 (2), p. 190-191, 193.

BLom Frans y Gertrude Duby

1955-1957 La selva Lacandona, Editorial Cultura, México, 2 vols.

BLOM Frans y Oliver LAFARGE

1926-1927 Tribes and Temples, Tulane University, New Orleans.

BRETON Alain

1979 Les Tzeltal de Bachajón. Habitat et organisation sociale, Laboratoire d'ethnologie (Recherches américaines, 3), Nanterre.

CARrasco Ramón y Andreé Bojalil

2005 "Nuevos datos para la historia del arte y la iconografía del Clásico temprano en el área maya: el Reino de Ka'an”, La pintura mural prehispánica en México. Boletín Informativo, 23, p. 24-32.

Culbert Patrick T.

1965 The ceramic history of the Central Highlands of Chiapas, Mexico, New World Archaeological Foundation, Brigham Young University (Papers of the New World Archaeological Foundation, 19), Provo.

Culbert Patrick T. y Don S. Rice

1990 Precolumbian population history in the Maya lowlands, University of New Mexico Press, Albuquerque.

Demarest Arthur A., Prudence M. Rice y Don S. Rice

2004 The Terminal Classic in the Maya lowlands. Collapse, transition and transformation, University Press of Colorado, Boulder.

EкHоLм Susana

1973 The Olmec rock carving at Xoc, Chiapas, Mexico, New World Archaeological Foundation, Brigham Young University (Papers of the New World Archaeological Foundation, 32), Provo.

Forné Mélanie, Ronald L. Bishop, Arthur A. Demarest, James M. Blackman y Erin L. SEARS

2010 "Gris Fino, Naranja Fino: presencia temprana y fuentes de producción, el caso de Cancuen", in Bárbara Arroyo, Adriana Linares Palma, Lorena Paiz Aragón y Ana Lucía Arroyave (eds), XXIII Simposio de investigaciones arqueológicas en Guatemala, 2009, Ministerio de Cultura y Deportes/Museo Nacional de Arqueología y Etnología/Instituto de Antropología e Historia/Asociación Tikal, Guatemala, vol. 1, p. 1163-1182.

Forsyth Donald W.

2005 "A survey of Terminal Classic ceramic complexes and their socioeconomic implications", in Sandra L. López Varela y Antonia E. Foias (eds), Geographies of power. Understanding the nature of Terminal Classic pottery in the Maya lowlands, Archaeopress (BAR International Series, 1447), Oxford, p. 7-22.

García Moll Roberto

2005 Pomoná: un sitio del Clásico maya en las colinas tabasqueñas, Instituto Nacional de Antropología e Historia (Colección Científica, 481), México.

Golden Charles y Andrew K. Scherer

2013 "Territory, trust, growth, and collapse in Classic Period Maya kingdoms", Current Anthropology, 54 (4), p. 397-435. 
Golden Charles, Andrew K. Scherer, René Muñoz y Zachary Hruby

2012 "Polities, boundaries and trade in the Classic Period Usumacinta river basin", Mexicon, 34 (1), p. 11-19.

GRAHAM Ian

2006 Corpus of Maya hieroglyphic inscriptions, vol. 9, part 2. Tonina, Peabody Museum of Archaeology and Ethnology, Harvard University, Cambridge (MA).

Graham Ian y Peter L. Mathews

1996 Corpus of Maya hieroglyphic inscriptions, vol. 6, part 2. Tonina, Peabody Museum of Archaeology and Ethnology, Harvard University, Cambridge (MA).

1999 Corpus of Maya hieroglyphic inscriptions, vol. 6, part 3. Tonina, Peabody Museum of Archaeology and Ethnology, Harvard University, Cambridge (MA).

LACADENA Alfonso

1996 “A new proposal for the transcription of the a-k'u-na/a-k'u-HUN-na title", Mayab, 10, p. 46-49.

Laló JaCinto Gabriel y María de la Luz Aguilar

1993 "El proyecto arqueológico Tenam Puente", Cuarto Foro de Arqueología de Chiapas, Gobierno del Estado de Chiapas, Tuxtla Gutiérrez, p. 151-161.

Liendo Stuardo Rodrigo

2001 "El paisaje urbano de Palenque: una perspectiva regional", Anales de Antropología 35, p. 213-231.

Marcus Joyce

1976 Emblem and State in the Classic Maya Lowlands: an epigraphical approach to theorical organization, Dumbarton Oaks, Washington (DC).

MARTin Simon y Nikolaï GruBE

2000 Chronicle of the Maya Kings and Queens. Deciphering the dynasties of the ancient Mayas, Thames and Hudson, London.

Mathews Peter L.

1983 Corpus of Maya hieroglyphic inscriptions, vol. 6, part 1. Tonina, Peabody Museum of Archaeology and Ethnology, Harvard University, Cambridge (MA).

1997 La escultura de Yaxchilán, INAH (Colección Científica, 368), México.

2001 "The dates of Tonina and a Dark Horse in its History", The PARI Journal, 2 (1), p. 1-5.

MAYER Karl H.

1980 Maya monuments. Sculptures of unkown provenance in the United States, Acoma Books, California.

1984 Maya monuments. Sculptures of unkown provenance in Middle America, Verlag Karl-Friedrich von Flemming, Berlin.

2007a "An unpublished Maya statue with glyphs from Tonina, Chiapas, México", Mexicon, 29 (6), p. 134-137.

2007b "An unpublished Maya inscribed sculpture from Tonina, Chiapas, México", Mexicon, 29 (1), p. 16-17.

MuÑoz Arturo René

2006 La secuencia cerámica de Piedras Negras, Guatemala: tipos y variedades [en línea], suplemento del informe Las cerámicas de Piedras Negras, Famsi, http://www.famsi.org/reports/02055es/, consultado el 05/12/2017. 
Palacios Enrique Juan

1928 En los confines de la Selva Lacandona. Exploraciones en el estado de Chiapas, mayo-agosto 1926, contribución de México al XXIII Congreso de Americanistas, Secretaria de Ediucación Pública y Talleres Gráficos de la Nación, México.

Paris Elizabeth H., Eric Taladoire y Thomas Arvol LeE Whiting

2013 "Estatus, poder y arquitectura del paisaje en el Centro Monumental de Moxviquil, Chiapas, México", in Víctor Manuel Esponda Jimeno (ed.), Anuario del Centro de Estudios Superiores de México y Centroamérica 2011, Universidad de Ciencias y Artes de Chiapas, Tuxtla Gutiérrez, p. 13-48.

2015 "Return to Moxviquil: new investigations and old collections", Ancient Mesoamerica, 26 (1), p. 81-112.

Patrois Julie

2000 Les sculptures en pierre de Tonina (Chiapas, Mexique), mémoire de DEA, UMR 8096 "Archéologie des Amériques", CNRS/Université de Paris 1, Paris.

Pescador CANTÓn Laura

2006 “Tonina”, in Roberto García Moll, Laura Pescador Cantón, Hernando Gómez Rueda, Antonio Benavides Castillo, Erik Velásquez García y Sonia E. Rivero Torres, Mayas, Zoques et autres culturess. Le sud-est mexicain : le Chiapas, INAH (Cités précolombiennes. Étude et reconstruction, 2), México, p. 38-47.

RANDS Robert L.

1967 “Cerámica de la región de Palenque, México", Estudios de Cultura Maya, 6, p. 111-147.

Rands Robert L., Ronald L. Bishop y Jeremy A. SABLOFF

1982 "Maya fine paste ceramics: an archaeological perspective", in Jeremy A. Sabloff (ed.), Excavations at Seibal, Department of Petén, Guatemala. Analysis of fine paste ceramics, Harvard University (Memoirs of the Peabody Museum of Archaeology and Ethnology, vol. 15, n. ${ }^{\circ}$ 2), Cambridge, p. 315-338.

ScARborough Vernon L.

1991 "Late preclassic ball courts at Cerros", in David A. Freidel (ed.), Archaeology at Cerros, Belize, Central America, vol. 3. The settlement system in a Late Preclassic Maya community, Southern Methodist University Press, Dallas, p. 107-125.

SCHELE Linda

1991 "An epigraphic history of the western maya lowlands", in Patrick T. Culbert (ed.), Classic Maya political history: hieroglyphic and archaeological evidence, Cambridge University Press (School of American Research, advanced seminar series), Cambridge, p. 72-101.

Sheseña Hernández Alejandro y Alejandro Tovalín-Ahumada

2014 "Los sitios arqueológicos de Chilón, Náchoj, Mukana y Nátentsun, municipio de Chilón, Chiapas”, LiminaR [en línea], 12 (1), p. 53-64, http://www.scielo. org.mx/scielo.php?script=sci_arttext\&pid=S1665-80272014000100004, consultado el 05/12/2017.

Stuart David

2011 "New Captive Sculptures from Tonina", Maya Decipherment [en línea], https:// decipherment.wordpress.com/2011/07/07/new-captive-sculptures-from-tonina/, consultado el 05/12/2017. 
TALADOIRE Eric

1980 “Réévaluation d'un des critères de définition des centres cérémoniels majeurs dans les Basses Terres Mayas", Boletin, 2, p. 39-53.

2015 "Towards a reevaluation of the Tonina polity", Estudios de Cultura Maya, 46, p. 45-70.

2016 "Las bases económicas de una entidad política maya. El caso de Toniná", Estudios de Cultura Maya, 48, p. 11-37.

THOMPson J. Eric S.

1944 Field notes, ms., Na Bolom Library, San Cristobal de las Casas.

Walling Stanley, Christine Taylor, Travis Cornish, Iakov Doumanoff, Chance CoughenOuR y Katharine LuKach

2012 "The commoner Ballcourt at Chawak But'o'ob, Belize: myth, ritual, and hydrology at a rural Maya site", paper presented at the Annual meeting of the Society for American Archaeology, Memphis, Tennessee.

Wichmann Soren y Alfonso LaCADENA

2005 "The dynamics of language in the Western Lowland Maya region", in Andrea Waters-Rist, Christine Cluney, Calla McNamee y Larry Steinbrenner (eds), Art for Archaeology's sake. Material culture and style across the disciplines, proceedings of the $33^{\text {rd }}$ Annual Chacmool Conference, Archaeological Association of the University of Calgary, Calgary (Canada), p. 32-48.

YAdEUn Angulo Juan

1992 Tonina. El laberinto del inframundo, Espejo de Obsidiana, Gobierno del Estado de Chiapas, Tuxtla Gútierrez.

1993 Tonina, El Equilibrista, México. 
